\title{
Non-model-based Damage Identification of Plates Using Principal, Mean and Gaussian Curvature Mode Shapes
}

\author{
Y.F. Xu, W.D. Zhu*, S.A. Smith \\ Department of Mechanical Engineering, University of Maryland Baltimore County, 1000 \\ Hilltop Circle, Baltimore, MD 21250, USA
}

\begin{abstract}
Mode shapes have been extensively used to identify structural damage. This paper presents a new non-model-based method that uses principal, mean and Gaussian curvature mode shapes (CMSs) to identify damage in plates; the method is applicable and robust to mode shapes associated with low and high elastic modes on dense and coarse measurement grids. A multiscale discrete differential-geometry scheme is proposed to calculate principal, mean and Gaussian CMSs associated with a mode shape of a plate, which can alleviate adverse effects of measurement noise on calculating the CMSs. Principal, mean and Gaussian CMSs of a damaged plate and those of an undamaged one are used to yield four curvature damage indices (CDIs), including Maximum-CDIs, Minimum-CDIs, Mean-CDIs and Gaussian-CDIs. Damage can be identified near regions with consistently higher values of the CDIs. It is shown that a mode shape of an undamaged plate can be well approximated using a polynomial of a properly determined order that fits
\end{abstract}

\footnotetext{
*Corresponding author. Tel: +1 410455 3394; fax: +1 4104551052.

Email addresses: yxu2@umbc.edu (Y.F. Xu), wzhu@umbc.edu (W.D. Zhu), ssmith11@umbc.edu (S.A. Smith)
} 
a mode shape of a damaged one, provided that the undamaged plate has a smooth geometry and is made of material that has no stiffness and mass discontinuities. Fitting and convergence indices are introduced to quantify the level of approximation of a mode shape from a polynomial fit to that of a damaged plate and to determine the proper order of the polynomial fit, respectively. A weight function is applied to the proposed CDIs to alleviate adverse effects of measurement noise on the CDIs and manifest existence of damage in the CDIs. A mode shape of an aluminum plate with damage in the form of a machined thickness reduction area was measured to experimentally investigate effectiveness of the proposed CDIs in damage identification; the damage on the plate was successfully identified. The experimental damage identification results were numerically verified by applying the proposed method to the mode shape associated with the same mode as that of the measured one from a finite element model of the damaged plate.

\section{Introduction}

Vibration-based damage detection has been a major research topic of structural dynamics in the past few decades. Measured modal characteristics, such as natural frequencies and mode shapes, are processed in various methods for detecting, locating and characterizing damage in structures, since modal characteristics are related to physical properties of structures, such as mass, stiffness and damping, which can change due to damage. A method can be categorized as a model-based or non-model-based method; the difference between them is that the former requires use of an accurate model of a structure and the latter does not. A method that only requires a min- 
imum amount of measured natural frequencies was developed to accurately detect locations and extent of damage in such structures as lightening masts $[1,2]$, space frames [3] and pipelines [4]. It is model-based and requires an accurate physics-based model of a structure, and effectiveness of the method highly depend on accuracy of the model of the structure. However, it can be difficult to construct models of most structures that can accurately predict their natural frequencies before and after occurrence of damage.

Methods that use measured mode shapes to identify damage in a structure can be good alternatives. While effects of damage on natural frequencies are global, those on mode shapes are local; abrupt changes in mode shapes in the neighborhood of damage can be observed. A two-dimensional gapped smoothing method was developed based on a one-dimensional gapped smoothing method [5]. Curvature mode shapes (CMSs) and curvatures of operating deflection shapes were used in the two-dimensional method to identify damage in plates [6], where mode shapes of an undamaged plate were not needed. A gapped polynomial fitting the curvatures was used to eliminate global trends of CMSs and curvatures of operating deflection shapes at each measurement point. A method that used curvatures of frequency-shift surfaces of plates to identify damage was proposed in Ref. [7]; curvatures of frequency-shift surfaces of associated undamaged plates could be obtained using a technique of locally weighted regression. It was shown to be better than the two-dimensional gapped smoothing method, since a frequencyshift surface contained information of a squared mode shape. A CMS-based method was proposed in Ref. [8], where a CMS of a plate based on an average curvature was calculated using a wavelet transform to alleviate adverse 
effects of measurement noise on the CMS and a Teager energy operator was applied to the CMS at each measurement point to eliminate the global trend of the transformed CMS. The mode shape-based methods mentioned above are local ones, and their common disadvantage is that they can be computationally inefficient, especially for a large and dense measurement grid, since the global trend of a curvature is locally eliminated in a point-by-point manner. A simplified gapped smoothing method, a generalized fractal dimension method and a strain energy method were used to detect delamination in a composite plate [9], and the methods there are also local ones and can be computationally inefficient. Changes in curvatures of uniform-load surfaces were used to identify damage in plates; the curvatures were shown to be sensitive to presence of local damage, even with truncated, incomplete and noisy measurements [10]. The method in Ref. [10] used natural frequencies and mode shapes of the first few modes of damaged and undamaged plates, but those of undamaged plates can be unavailable in practice. Mean and Gaussian curvature shapes of three-dimensional digital models of structures obtained by a terrestrial laser scanner were used to identify mass loss of concrete via piecewise comparisons of distributions of the curvature shapes [11]. A limitation of the method is that only surface damage can be identified. Besides curvature-based methods, wavelet transform-based methods have been widely studied to identify damage in plates $[12,13,14]$. Gabor wavelets were used to identify damage in a rectangular plate [12]; effects of various wavelets on identifying damage, such as Haar, Daubechies, Gaussian and Coiflet wavelets, were studied and compared in Ref. [13]. Depths of cracks in plates could be detected using a wavelet transform-based method 
with the aid of models of undamaged plates [14]. However, whether damage can be identified using a wavelet transform-based method depends on the type and parameters of an applied wavelet. Changes in the strain energy of a structure have been used to identify damage; the method was extended from the one for beams [15], which require mode shapes of damaged and undamaged structures. A two-dimensional polynomial annihilation edge detection method was proposed for detection and localization of damage in plates [16]; it was extended from the one for beams, which can detect discontinuities in piecewise smooth functions and their derivatives [17]. The limitation of the method is that only edges of damage could be identified.

A non-model-based method based on principal, mean and Gaussian CMSs is proposed in this work to identify damage in plates. Theoretical bases of principal CMSs of a plate are shown. A multi-scale discrete differentialgeometry scheme is proposed to calculate principal, mean and Gaussian CMSs associated with a mode shape of a plate, which can alleviate adverse effects of measurement noise on calculating the CMSs. Principal CMSs are directly related to principal stresses of a deformed plate, and mean and Gaussian CMSs can quantify differential-geometry features of a mode shape of the plate. Differences between principal, mean and Gaussian CMSs of a damaged plate and those of the associated undamaged one are used to yield four curvature damage indices (CDIs), including Maximum-CDI, Minimum-CDI, Mean-CDI and Gaussian-CDI. Global trends of the CMSs are eliminated in a global manner and can be computationally more efficient than the local methods. A mode shape from a polynomial of a properly determined order that fits a mode shape of a damaged plate can be used to approximate the 
corresponding mode shape of the associated undamaged one, provided that the undamaged plate has a smooth geometry and is made of material that has no stiffness and mass discontinuities. Fitting and convergence indices are introduced to assist determination of the proper order of the polynomial fit. A weight function is applied to the proposed CDIs to alleviate adverse effects of measurement noise on the CDIs and manifest existence of damage in the CDIs. The applicability and robustness of the proposed method to a mode shape of a low elastic mode on a coarse measurement grid are numerically investigated. An aluminum plate with damage in the form of a machined thickness reduction area was constructed, and a mode shape of the damaged plate was measured using non-contact excitation and measurement to investigate effectiveness of the proposed method. The mode shape associated with the same mode as that of the measured mode shape from a finite element model of the damaged plate was used to numerically verify the experimental damage identification results.

\section{Methodology}

A finite element model of a damaged rectangular steel plate that has a length of $0.3 \mathrm{~m}$, a width of $0.4 \mathrm{~m}$ and a thickness of $0.002 \mathrm{~m}$ is constructed using commercial finite element software ABAQUS; the model has a total of $150 \times 200$ square plate elements. The damage is in the form of a thickness reduction area, and its position and dimensions are shown in Fig. 1(a); the depth of the thickness reduction area is $0.0002 \mathrm{~m}$. The mass density, elastic modulus and Poisson's ratio of the plate are $7850 \mathrm{~kg} / \mathrm{m}^{3}, 200 \mathrm{GPa}$ and 0.3, respectively. A finite element model of an undamaged plate of the same 
dimensions and material properties as those of the damaged plate is also constructed, which has the same number of square plate elements as that of the damaged one. To demonstrate the proposed method, undamped mode shapes of the damaged and undamaged plates associated with their 23-rd elastic modes are calculated and denoted by $\mathbf{Z}^{d, 23}$ and $\mathbf{Z}^{u, 23}$, respectively; $\mathbf{Z}^{d, 23}$ and $\mathbf{Z}^{u, 23}$ are in the same phase, and they are normalized so that their maximum absolute values are equal to one. Mode shapes $\mathbf{Z}^{d, 23}$ and $\mathbf{Z}^{u, 23}$ are shown in Figs. 1(b) and (c), respectively.

\subsection{Principal CMSs of plates}

While the curvature at a point on a mode shape of a one-dimensional structure, such as a beam, is defined along the length of the structure, that on a mode shape of a plate is defined on a unit vector tangent to the mode shape at the point, and its value depends on not only the mode shape but the unit vector. The curvature at a point $\mathbf{p}$ on a mode shape $\mathbf{Z}$ shown in Fig. 2 with respect to a unit vector $\mathbf{v}$ tangent to $\mathbf{Z}$ at $\mathbf{p}$ is defined by [18]

$$
\kappa_{\mathbf{Z}, \mathbf{p}}(\mathbf{v})=-\left(\nabla_{\mathbf{v}} \mathbf{n}\right) \cdot \mathbf{v}
$$

where $\mathbf{n}$ is a unit normal vector field in the neighborhood of $\mathbf{p}$ on $\mathbf{Z}$, and $\nabla_{\mathbf{v}} \mathbf{n}$ is the covariant derivative of $\mathbf{n}$ with respect to $\mathbf{v}$, which can be defined by

$$
\nabla_{\mathbf{v}} \mathbf{n}=\left.\frac{\mathrm{d}}{\mathrm{d} t} \mathbf{n}(\mathbf{p}+t \mathbf{v})\right|_{t=0}
$$

The geometric meaning of the curvature is that it quantifies the bending rate of a curve $\sigma$ on $\mathbf{Z}$ with respect to $\mathbf{n} ; \sigma$ is obtained by intersecting $\mathbf{Z}$ with a 
plane that is determined by $\mathbf{n}$ and $\mathbf{v}$, as shown in Fig. 2. When $\mathbf{p}$ is an umbilic point, $\kappa_{\mathbf{Z}, \mathbf{p}}$ is equal to one value with any $\mathbf{v}$. Examples of surfaces that consist of umbilic points are planes and spheres. More commonly for a mode shape, when $\mathbf{p}$ is a nonumbilic point, there are two orthogonal principal directions, along which $\kappa_{\mathbf{Z}, \mathbf{p}}$ attains its maximum and minimum values, denoted by $\kappa_{\mathbf{Z}, \mathbf{p}}^{\max }$ and $\kappa_{\mathbf{Z}, \mathbf{p}}^{\min }$, respectively; the two curvatures, i.e., the maximum and minimum curvatures, are termed as principal curvatures. Shapes that are formed by maximum and minimum curvatures associated with a mode shape are termed as maximum and minimum CMSs, respectively, and they are principal CMSs.

For a constant-thickness plate made of homogeneous material, one has $[19]$

$$
\left[\begin{array}{c}
M_{x x} \\
M_{y y} \\
M_{x y}
\end{array}\right]=\frac{E h^{3}}{12\left(\nu^{2}-1\right)}\left[\begin{array}{ccc}
1 & \nu & 0 \\
\nu & 1 & 0 \\
0 & 0 & \nu-1
\end{array}\right]\left[\begin{array}{c}
\kappa_{x x} \\
\kappa_{y y} \\
\kappa_{x y}
\end{array}\right]
$$

where $M_{x x}$ and $M_{y y}$ are bending moments per unit length acting on edges of a differential element parallel to $y$ - and $x$-axes of a global three-dimensional Cartesian coordinate $O-x y z$, respectively; $M_{x y}$ is the twisting moment with respect to $x$ - and $y$-axes; $E, h$ and $\nu$ are the Young's modulus, thickness and Poisson's ratio of the plate, respectively; $\kappa_{x x}$ and $\kappa_{y y}$ are curvatures with respect to $x$ - and $y$-axes, respectively; and $\kappa_{x y}$ is the twist with respect to $x$ and $y$-axes. For a point $\mathbf{p}$ on the plate, a local Cartesian coordinate $\mathbf{p}-x^{\prime} y^{\prime} z^{\prime}$ can be defined, where $x^{\prime}$ - and $y^{\prime}$-axes are along principal directions associated with the maximum and minimum curvatures of $\mathbf{p}$ on $\mathbf{Z}$, respectively. In this case, associated $M_{x^{\prime} y^{\prime}}$ and $\kappa_{x^{\prime} y^{\prime}}$ vanish, and one has 


$$
\left\{\begin{array}{l}
\kappa_{\mathbf{Z}, \mathbf{p}}^{\mathrm{Max}}=-\frac{12}{E h^{3}}\left(M_{x^{\prime} x^{\prime}}-\nu M_{y^{\prime} y^{\prime}}\right) \\
\kappa_{\mathbf{Z}, \mathbf{p}}^{\mathrm{Min}}=-\frac{12}{E h^{3}}\left(M_{y^{\prime} y^{\prime}}-\nu M_{x^{\prime} x^{\prime}}\right)
\end{array}\right.
$$

Principal curvatures can be used to construct a quadratic approximation of $\mathbf{Z}$ near p. When $\mathbf{v}$ in Eq. (1) is not on a principal direction, $\kappa_{\mathbf{Z}, \mathbf{p}}$ can be expressed by [18]

$$
\kappa_{\mathbf{Z}, \mathbf{p}}(\mathbf{v})=\kappa_{\mathbf{Z}, \mathbf{p}}^{\mathrm{Max}} \cos ^{2} \vartheta_{1}+\kappa_{\mathbf{Z}, \mathbf{p}}^{\mathrm{Min}} \cos ^{2} \vartheta_{2}
$$

where $\vartheta_{1}$ and $\vartheta_{2}$ are angles between $\mathbf{v}$ and principal directions associated with the maximum and minimum curvatures, respectively. Hence, principal curvatures can be considered to be properties of $\mathbf{Z}$ at $\mathbf{p}$ that are independent of $\mathbf{v}$.

\subsection{Multi-scale discrete differential-geometry scheme}

When $\mathbf{Z}$ can be analytically expressed, principal CMSs can be calculated using a well-established analytical method [18]. When $\mathbf{Z}$ cannot be analytically expressed and is presented in a discrete form, a numerical scheme is needed to calculate discrete principal CMSs associated with Z Z. For a onedimensional structure, a discrete CMS can be accurately calculated using a finite difference scheme. Discrete principal CMSs associated with $\mathbf{Z}$ cannot be easily calculated in that $\kappa_{\mathbf{Z}, \mathbf{p}}$ depends on $\mathbf{v}$, as shown in Eq. (1), and there are an infinite number of $\mathbf{v}$ that can be defined at $\mathbf{p}$. One can perform a one-dimensional finite difference scheme at $\mathbf{p}$ with respect to different $\mathbf{v}$ to calculate curvatures and find principal curvatures as the maximum and minimum values among resulting curvatures, but it can be computationally inefficient. 
To efficiently and accurately calculate principal CMSs associated with Z , two operators in Ref. [20] for shapes with a triangulated mesh are introduced; they can be used to calculate its mean and Gaussian curvatures at a point $\mathbf{p}$, denoted by $H_{\mathbf{Z}, \mathbf{p}}$ and $G_{\mathbf{Z}, \mathbf{p}}$, respectively. Mean and Gaussian curvatures of $\mathbf{Z}$ at $\mathbf{p}$ are the average and product of two principal curvatures of $\mathbf{Z}$ at $\mathbf{p}$, respectively, which can be expressed by

$$
H_{\mathbf{Z}, \mathbf{p}}=\frac{\kappa_{\mathbf{Z}, \mathbf{p}}^{\mathrm{Max}}+\kappa_{\mathbf{Z}, \mathbf{p}}^{\mathrm{Min}}}{2}
$$

and

$$
G_{\mathbf{Z}, \mathbf{p}}=\kappa_{\mathbf{Z}, \mathbf{p}}^{\mathrm{Max}} \times \kappa_{\mathbf{Z}, \mathbf{p}}^{\mathrm{Min}}
$$

Shapes that are formed by mean and Gaussian curvatures associated with a mode shape are termed as mean and Gaussian CMSs, respectively. In the operators for mean and Gaussian curvatures of $\mathbf{p}$ on $\mathbf{Z}$ with a triangulated mesh, a new surface area $A_{\mathbf{P}}$ is calculated for a one-ring neighborhood associated with $\mathbf{p}$, which is formed by all triangulated elements of the mesh consisting of $\mathbf{p}$, as shown in Fig. 3(a). The pseudo-code for calculating $A_{\mathbf{P}}$ is shown in Fig. 4, where the Voronoi area of an acute triangle $\triangle \mathbf{p q r}$ shown in Fig. 3(b) can be expressed by

$$
A_{\text {Voronoi }}=\frac{1}{8}\left(|\mathbf{p r}|^{2} \cot \angle \mathbf{q}+|\mathbf{p q}|^{2} \cot \angle \mathbf{r}\right)
$$

and $A$ is the area of a triangle in the one-ring neighborhood. Mean and Gaussian curvatures of $\mathbf{p}$ on $\mathbf{Z}$ can be calculated by 


$$
H_{\mathbf{Z}, \mathbf{p}}=\frac{1}{4 A_{\mathbf{P}}}\left[\sum_{i \in N_{1}(\mathbf{p})}\left(\cot \alpha_{i}+\cot \beta_{i}\right)\left(\mathbf{p}-\mathbf{q}_{i}\right)\right] \cdot \mathbf{n}(\mathbf{p})
$$

and

$$
G_{\mathbf{Z}, \mathbf{p}}=\frac{2 \pi-\sum_{i \in N_{1}(\mathbf{p})} \theta_{i}}{A_{\mathbf{P}}}
$$

where $N_{1}(\mathbf{p})$ is the number of points that are connected with $\mathbf{p}$ in the one-ring neighborhood, and $\theta_{i}$ is the angle associated with $\mathbf{q}_{i}$, as shown in Fig. 3(a). With calculated $H_{\mathbf{Z}, \mathbf{p}}$ and $G_{\mathbf{Z}, \mathbf{p}}$, principal curvatures at $\mathbf{p}$ can be calculated by

$$
\kappa_{\mathbf{Z}, \mathbf{p}}^{\operatorname{Max}}=H_{\mathbf{Z}, \mathbf{p}}+\sqrt{H_{\mathbf{Z}, \mathbf{p}}^{2}-G_{\mathbf{Z}, \mathbf{p}}}
$$

and

$$
\kappa_{\mathbf{Z}, \mathbf{p}}^{\mathrm{Min}}=H_{\mathbf{Z}, \mathbf{p}}-\sqrt{H_{\mathbf{Z}, \mathbf{p}}^{2}-G_{\mathbf{Z}, \mathbf{p}}}
$$

Similar to calculating curvatures of a one-dimensional structure [21], those associated with a mode shape of a plate can be contaminated by measurement noise. To alleviate adverse effects of measurement noise on calculating curvatures of a plate, a multi-scale discrete differential-geometry scheme is proposed based on the operators introduced above, where a hexagonal onering neighborhood is constructed at each measurement point. The hexagonal one-ring neighborhood associated with $\mathbf{p}$ projected onto the undeformed plate is equilateral with a side length of $s$, as shown in Fig. 3(c). Since $x y$ coordinates of $\mathbf{q}_{i}$, where $i=1,2, \ldots, 6$, can be analytically obtained from the geometry of the hexagonal one-ring neighborhood, its $z$-coordinate can 
be obtained from interpolation based on $\mathbf{Z}$. CMSs of $\mathbf{p}$ can be obtained based on the hexagonal one-ring neighborhood, and $s$ is considered as the scale of the scheme. For a measured mode shape with an unknown noise level, one needs to progressively test different values of $s$ from smaller to larger ones. A proper value of $s$ is the one with which the resulting CMS becomes smooth and has a clear global trend.

To illustrate adverse effects of measurement noise and the effectiveness of the scheme, white noise is added to $\mathbf{Z}^{d, 23}$ with a signal-to-noise ratio of 60 $\mathrm{db}$ to simulate measurement noise. Maximum CMSs associated with $\mathbf{Z}^{d, 23}$ from the scheme with $s=0.002 \mathrm{~m}, 0.005 \mathrm{~m}$ and $0.015 \mathrm{~m}$ are shown in Fig. 5(a) through (c), respectively; the maximum CMS associated with noisefree $\mathbf{Z}^{d, 23}$ is shown in Fig. 5(d). It can be seen that measurement noise is amplified and becomes dominant in the resulting maximum CMS from the scheme with $s=0.002 \mathrm{~m}$, since differences between the value of a noise-free $\mathbf{Z}^{d, 23}$ at a point and those in the hexagonal one-ring neighborhood with a side length of $s$ are small compared with those associated with $\mathbf{Z}^{d, 23}$ with the measurement noise. Figures 5(b) and (c) show that maximum CMSs can be obtained with a lower noise level with a larger value of $s$. While maximum curvatures of $\mathbf{Z}^{d, 23}$ at a point from the scheme with different values of $s$ are different from those associated with noise-free $\mathbf{Z}^{d, 23}$ at the point from the scheme with $s=0.002 \mathrm{~m}$, global trends of the maximum CMSs are retained; the larger the value of $s$, the lower the noise level in the resulting maximum CMS, which is also the case for minimum, mean and Gaussian CMSs. 


\subsection{CMS-based damage indices}

CMSs have been widely used to identify damage in beams, since the curvature at a point on a beam can be expressed by [22]

$$
v^{\prime \prime}=\frac{M}{E I}
$$

where $v^{\prime \prime}$ is the curvature at the point, $M$ is the bending moment applied at the point, and $E I$ is the bending stiffness of the cross-section at the point. Since damage can introduce reduction in $E I$ in its neighborhood, the magnitude of $v^{\prime \prime}$ increases in the neighborhood of the damage compared with that of the undamaged beam. Differences between $v^{\prime \prime}$ associated with the damaged and undamaged beams can be used for identifying the damage. When $E$ in Eq. (4) changes in the neighborhood of damage in a plate, magnitudes of principal curvatures in the neighborhood of the damage would change, and so would those of mean and Gaussian curvatures. Hence, differences between principal, mean and Gaussian CMSs associated with the damaged and undamaged plates can be used for identifying damage in a plate. Four CDIs associated with a mode shape of a plate are proposed to identify damage; for each point on the plate, the CDIs are listed below:

1. Maximum-CDI. A Maximum-CDI denoted by $\delta^{\operatorname{Max}}(\mathbf{p})$ is defined by

$$
\delta^{\operatorname{Max}}(\mathbf{p})=\left(\kappa_{\mathbf{Z}^{d, j}, \mathbf{p}}^{\mathrm{Max}}-\kappa_{\mathbf{Z}^{u, j}, \mathbf{p}}^{\mathrm{Max}}\right)^{2}
$$

2. Minimum-CDI. A Minimum-CDI denoted by $\delta^{\text {Min }}(\mathbf{p})$ is defined by

$$
\delta^{\operatorname{Min}}(\mathbf{p})=\left(\kappa_{\mathbf{Z}^{d, j}, \mathbf{p}}^{\operatorname{Min}}-\kappa_{\mathbf{Z}^{u, j}, \mathbf{p}}^{\operatorname{Min}}\right)^{2}
$$

3. Mean-CDI. A Mean-CDI denoted by $\delta^{\text {Mean }}(\mathbf{p})$ is defined by 


$$
\delta^{\text {Mean }}(\mathbf{p})=\left(H_{\mathbf{Z}^{d, j}, \mathbf{p}}-H_{\mathbf{Z}^{u, j}, \mathbf{p}}\right)^{2}
$$

4. Gaussian-CDI. A Gaussian-CDI denoted by $\delta^{\text {Gaussian }}(\mathbf{p})$ is defined by

$$
\delta^{\text {Gaussian }}(\mathbf{p})=\left(G_{\mathbf{Z}^{d, j}, \mathbf{p}}-G_{\mathbf{Z}^{u, j}, \mathbf{p}}\right)^{2}
$$

Maximum-CDIs, Minimum-CDIs, Mean-CDIs and Gaussian-CDIs associated with noise-free $\mathbf{Z}^{d, 23}$ and $\mathbf{Z}^{u, 23}$ with $s=0.002 \mathrm{~m}$ in the scheme are shown in Fig. 6(a) through (d), respectively. It can be seen that the damage can be identified near regions with consistently higher values of the CDIs.

\subsection{Approximation of mode shapes of an undamaged plate}

While the CDIs proposed above can be used to identify damage in a plate, they require use of mode shapes of an undamaged plate, which are usually unavailable in practice. Assuming that existence of relatively small damage in a plate does not cause prominent changes in its mode shapes in the neighborhood of the damage, one can approximate mode shapes of the associated undamaged plate using polynomials that fit the corresponding mode shapes of the damaged plate, provided that the undamaged plate is geometrically smooth and made of materials that have no stiffness and mass discontinuities. The modal assurance criterion (MAC) value [23] in percent between $\mathbf{Z}^{d, 23}$ and $\mathbf{Z}^{u, 23}$ is $99.95 \%$, which indicates that they are almost identical to each other and validates the assumption on the existence of relatively small damage. A similar technique has been proposed in Ref. [21] to approximate mode shapes of an undamaged beam using polynomials that fit corresponding mode shapes of the damaged one. Mode shapes of an 
undamaged plate corresponding to those of a damaged one are not measured in this work, and it is proposed that a mode shape of an undamaged plate be obtained from a polynomial of a properly determined order that fits the corresponding mode shape of the damaged plate:

$$
z^{p}(x, y)=\sum_{k=0}^{n} \sum_{i=0}^{k} a_{i, k-i} x^{i} y^{k-i}
$$

where $n$ is the order of the polynomial, which controls the level of approximation of the polynomial fit to the mode shape of the damaged plate, $(x, y)$ are $x y$-coordinates of a point on an undeformed plate, and $a_{i, k-i}$ are coefficients of the polynomial that can be obtained by solving a linear equation

$$
\mathbf{V a}=\mathbf{z}
$$

in which $\mathbf{z}$ is the $N$-dimensional mode shape vector of the damaged plate to be fit, $\mathbf{V}$ is the $N \times\left(\sum_{p=1}^{n+1} p\right)$-dimensional bivariate Vandermonde matrix which can be expressed by

$$
\mathbf{V}=\left[\begin{array}{ccccccccc}
1 & x_{1} & y_{1} & \ldots & x_{1}^{n} & \ldots & x_{1}^{i} y_{1}^{n-i} & \ldots & y_{1}^{n} \\
1 & x_{2} & y_{2} & \ldots & x_{2}^{n} & \ldots & x_{2}^{i} y_{2}^{n-i} & \ldots & y_{2}^{n} \\
\vdots & \vdots & \vdots & \ddots & \vdots & \ddots & \vdots & \ddots & \vdots \\
1 & x_{N} & y_{N} & \ldots & x_{N}^{n} & \ldots & x_{N}^{i} y_{N}^{n-i} & \ldots & y_{N}^{n}
\end{array}\right]
$$

and $\mathbf{a}$ is the $\left(\sum_{p=1}^{n+1} p\right)$-dimensional coefficient vector which can be expressed by

$$
\mathbf{a}=\left[\begin{array}{lllllllll}
a_{0,0} & a_{1,0} & a_{0,1} & \ldots & a_{n, 0} & \ldots & a_{i, n-i} & \ldots & a_{0, n}
\end{array}\right]^{\mathrm{T}}
$$


Solving Eq. (19) for the coefficient vector is equivalent to solving an unconstrained least-squares problem $\min \frac{1}{2}\left\|\mathbf{V a}^{*}-\mathbf{z}\right\|^{2}$ for an optimum minimizer $\mathbf{a}^{*}[24]$, which is usually an over-determined problem, i.e., $N>\sum_{p=1}^{n+1} p$. A solution can be obtained using the singular-value decomposition of $\mathbf{V}$ [24], which gives

$$
\mathbf{V}=\mathbf{U}\left[\begin{array}{l}
\mathbf{S} \\
0
\end{array}\right] \mathbf{W}^{\mathrm{T}}
$$

where $\mathbf{U}$ and $\mathbf{W}$ are $N \times N$ and $\left(\sum_{p=1}^{n+1} p\right) \times\left(\sum_{p=1}^{n+1} p\right)$ orthogonal matrices, respectively, and $\mathbf{S}$ is a $\left(\sum_{p=1}^{n+1} p\right) \times\left(\sum_{p=1}^{n+1} p\right)$ diagonal matrix. An optimum minimizer $\mathbf{a}^{*}$ based on the singular-value decomposition of $\mathbf{V}$ can be obtained by

$$
\mathbf{a}^{*}=\mathbf{W S}^{-1} \mathbf{U}_{1}^{\mathrm{T}} \mathbf{z}
$$

where $\mathbf{U}_{1}$ is a matrix formed by the first $\sum_{p=1}^{n+1} p$ columns of $\mathbf{U}$. When $n$ in Eq. (18) becomes a large value, $\mathbf{S}$ can be ill-conditioned, which can result in a low level of approximation of the associated polynomial fit. To avoid ill-conditioning of $\mathbf{S}$, it is proposed that $x$ and $y$ in Eq. (18) be normalized using the "center and scale" technique [25] before formulation of the linear equation in Eq. (19). Normalized coordinates $\tilde{x}$ and $\tilde{y}$ can be expressed by

$$
\left\{\begin{array}{l}
\tilde{x}=\frac{2 x-2 \bar{x}}{l_{1}} \\
\tilde{y}=\frac{2 y-2 \bar{y}}{l_{2}}
\end{array}\right.
$$

where $\bar{x}$ and $\bar{y}$ are $x$ - and $y$-coordinates of the center point of the plate, respectively, and $l_{1}$ and $l_{2}$ are lengths of the plate along $x$ - and $y$-axes, respectively. 
An increase of $n$ in the polynomial fit in Eq. (18) can improve its level of approximation of the resulting mode shape to that to be fit. To quantify the level of approximation, a fitting index fit in percent, defined by

$$
\text { fit }(n)=\frac{\operatorname{RMS}(\mathbf{z})}{\operatorname{RMS}(\mathbf{z})+\operatorname{RMS}(\mathbf{e})} \times 100 \%
$$

is used, where RMS $(\cdot)$ denotes the root-mean-square value of a vector and $\mathbf{e}$ is the error vector between the mode shape to be fit and the corresponding one from the current polynomial fit, i.e., $\mathbf{e}=\mathbf{V a}^{*}-\mathbf{z}$. When the fitting index is close to $100 \%$, the mode shape from the current polynomial fit is almost identical to $\mathbf{z}$; the lower the fitting index, the lower the level of approximation of the mode shape from the current polynomial fit. Fitting indices fit associated with $\mathbf{Z}^{d, 23}$ for different $n$ are shown in Fig. 7(a). It can be seen in Fig. 7(b) that fit converges to a certain value as $n$ increases. To determine the proper order of a polynomial fit, a convergence index con for the polynomial fit with $n \geqslant 3$ is defined based on fit, which can be expressed by

$$
\operatorname{con}(n)=\text { fit }(n)-\text { fit }(n-2)
$$

Convergence indices con associated with $\mathbf{Z}^{d, 23}$ for different $n$ are shown in Fig. $7(\mathrm{c})$. It can be seen that when $n$ is larger than a certain value, con starts to decrease. When con is sufficiently small after its start of decrease, there is no significant improvement in the level of approximation of the polynomial fit. To determine a proper value of $n$, it is proposed that the value be the smallest one with which con is smaller than a prescribed threshold value. In this work, the prescribed threshold value for con is $0.50 \%$. Hence, the proper value of 
$n$ associated with $\mathbf{Z}^{d, 23}$ is determined to be 15 , with which con $=0.42 \%$, as shown in Fig. 7(d). Maximum-CDIs, Minimum-CDIs, Mean-CDIs and Gaussian-CDIs associated with noise-free $\mathbf{Z}^{d, 23}$ and the corresponding mode shape from the polynomial fit with $n=15$ are shown in Fig. 8(a) through (d), respectively. Similar to the CDIs associated with noise-free $\mathbf{Z}^{d, 23}$ and $\mathbf{Z}^{u, 23}$ in Fig. 6, the damage can be identified near regions with higher values of CDIs.

\subsection{Denoising of $C D I s$}

Use of CDIs to identify damage depends on calculation of CMSs, which are usually subject to measurement noise that can fuzz existence of damage in the CDIs. A suitable value of $s$ in the scheme can alleviate adverse effects of measurement noise. Since the noise level of a measured mode shape is usually unknown, one needs to progressively test different values of $s$ from smaller to larger ones until a proper one is obtained, with which the existence of the damage becomes prominent in associated CDIs. However, the process of seeking a proper value of $s$ can be time-consuming and inefficient. Figures 9(a) and (b) show Maximum-CDIs associated with $\mathbf{Z}^{d, 23}$ with measurement noise and the corresponding mode shape from the polynomial fit with $s=$ $0.002 \mathrm{~m}$ and $0.005 \mathrm{~m}$ in the scheme, respectively, but the damage cannot be identified due to the measurement noise. When $s=0.015 \mathrm{~m}$, the damage can be identified near regions of higher values of Maximum-CDIs, as shown in Fig. 9(c). To manifest the effects of damage on the proposed CDIs with a smaller $s$ in the scheme, a two-dimensional discrete weight function is applied to differences between CMSs before calculating the CDIs. The weight function for a mesh with square elements is expressed by 


$$
W_{M_{w}}\left(k_{1}, k_{2}\right)=\mathrm{e}^{-4\left[\left(\frac{k_{1}}{M_{w}}\right)^{2}+\left(\frac{k_{2}}{M_{w}}\right)^{2}\right]}
$$

where $M_{w}$ is the scale of the weight function; $k_{1}$ and $k_{2}$ are integer coordinates associated with $x$ - and $y$-axes of the weight function, respectively, and $k_{1}, k_{2} \in\left[-M_{w}, M_{w}\right]$. For a mesh with non-square elements, one can interpolate a mode shape on a mesh with square elements so that the weight function can have equal weights along $x$ - and $y$-axes. An interpolation can also be conducted to obtain a mode shape on a finer mesh with better spatial resolution in resulting CDIs. Weighted CDIs $\tilde{\delta}^{\text {Max }}, \tilde{\delta}^{\text {Min }}, \tilde{\delta}^{\text {Mean }}$ and $\tilde{\delta}^{\text {Gaussian }}$ at a point $\mathbf{p}$ on a plate, based on the CDIs in Eqs. (14) through (17), respectively, can be expressed by

$$
\tilde{\delta}^{\operatorname{Max}}(\mathbf{p})=\left\{\sum_{k_{1}=-M_{w}}^{M_{w}} \sum_{k_{2}=-M_{w}}^{M_{w}}\left[\left(\kappa_{\mathbf{Z}^{d, j}, \mathbf{p}_{k_{1}, k_{2}}}^{\mathrm{Max}}-\kappa_{\mathbf{Z}^{u, j}, \mathbf{p}_{k_{1}, k_{2}}}^{\mathrm{Max}}\right) \times W_{M_{w}}\left(k_{1}, k_{2}\right)\right]\right\}^{2}
$$

$$
\tilde{\delta}^{\operatorname{Min}}(\mathbf{p})=\left\{\sum_{k_{1}=-M_{w}}^{M_{w}} \sum_{k_{2}=-M_{w}}^{M_{w}}\left[\left(\kappa_{\mathbf{Z}^{d, j}, \mathbf{p}_{k_{1}, k_{2}}}^{\operatorname{Min}}-\kappa_{\mathbf{Z}^{u, j}, \mathbf{p}_{k_{1}, k_{2}}}^{\operatorname{Min}}\right) \times W_{M_{w}}\left(k_{1}, k_{2}\right)\right]\right\}^{2}
$$

$$
\tilde{\delta}^{\text {Mean }}(\mathbf{p})=\left\{\sum_{k_{1}=-M_{w}}^{M_{w}} \sum_{k_{2}=-M_{w}}^{M_{w}}\left[\left(H_{\mathbf{Z}^{d, j}, \mathbf{p}_{k_{1}, k_{2}}}-H_{\mathbf{Z}^{u, j}, \mathbf{p}_{k_{1}, k_{2}}}\right) \times W_{M_{w}}\left(k_{1}, k_{2}\right)\right]\right\}^{2}
$$

$$
\tilde{\delta}^{\text {Gaussian }}(\mathbf{p})=\left\{\sum_{k_{1}=-M_{w}}^{M_{w}} \sum_{k_{2}=-M_{w}}^{M_{w}}\left[\left(G_{\mathbf{Z}^{d, j}, \mathbf{p}_{k_{1}, k_{2}}}-G_{\mathbf{Z}^{u, j}, \mathbf{p}_{k_{1}, k_{2}}}\right) \times W_{M_{w}}\left(k_{1}, k_{2}\right)\right]\right\}^{2}
$$


where $\mathbf{p}_{k_{1}, k_{2}}$ is a point with $x y$-coordinates $\left(x_{\mathbf{p}}+k_{1} \Delta d, y_{\mathbf{p}}+k_{2} \Delta d\right)$, in which $x_{\mathbf{p}}$ and $y_{\mathbf{p}}$ are $x$ - and $y$-coordinates of $\mathbf{p}$, respectively, and $\Delta d$ is the side length of an element of the mesh. Applying the weight function with $M_{w}=7$, weighted Maximum-CDIs associated with $\mathbf{Z}^{d, 23}$ and the corresponding mode shape from the polynomial fit with $s=0.002 \mathrm{~m}, 0.005 \mathrm{~m}$ and $0.015 \mathrm{~m}$ in the scheme are shown in Fig. 9(d) through (f), respectively. When $s=0.002 \mathrm{~m}$, the damage cannot be identified in the weighted Maximum-CDIs; however, when $s=0.005 \mathrm{~m}$, the damage can be identified near the region with higher values of Maximum-CDIs, and some disturbing regions with relatively high values of Maximum-CDIs can also be observed. When $s=0.015 \mathrm{~m}$, the damage can be identified with smaller disturbing regions with lower values of CDIs.

\subsection{Applicability and robustness of the method}

The applicability and robustness of the proposed method for identifying damage using a mode shape associated with a low elastic mode are numerically investigated here; use of the mode shape on a coarse measurement grid and that of the weight function for the mode shape with high and low signal-to-noise ratios are also discussed. Undamped mode shapes of the damaged and undamaged plates associated with their second elastic modes are calculated and denoted by $\mathbf{Z}^{d, 2}$ and $\mathbf{Z}^{u, 2}$, respectively; $\mathbf{Z}^{d, 2}$ and $\mathbf{Z}^{u, 2}$ are in the same phase, and they are normalized so that their maximum absolute values are equal to one, as shown in Fig. 10(a) and (b), respectively. The MAC value in percent between $\mathbf{Z}^{d, 2}$ and $\mathbf{Z}^{u, 2}$ is $99.9998 \%$, which indicates that they are almost identical to each other and validates the assumption on the existence of relatively small damage again. Fitting indices and con- 
vergence indices associated with $\mathbf{Z}^{d, 2}$ are shown in Figs. 11, and the proper value of $n$ associated with $\mathbf{Z}^{d, 2}$ is determined to be 8 . The mode shape from the polynomial fit with $n=8$, denoted by $\mathbf{Z}^{p, 2}$, is shown in Fig. $10(\mathrm{c})$; the MAC value in percent between $\mathbf{Z}^{d, 2}$ and $\mathbf{Z}^{p, 2}$ is almost $100 \%$.

Maximum-CDIs, Minimum-CDIs, Mean-CDIs and Gaussian-CDIs associated with noise-free $\mathbf{Z}^{d, 2}$ and $\mathbf{Z}^{u, 2}$ with $s=0.002 \mathrm{~m}$ in the scheme are shown in Figs. 12(a) through (d), respectively. The damage can be identified near regions with consistently higher values of the Maximum-CDIs, MinimumCDIs and Mean-CDIs. Relatively high values of the Gaussian-CDIs can be observed beyond the damage, near its edges. Maximum-CDIs, MinimumCDIs, Mean-CDIs and Gaussian-CDIs associated with noise-free $\mathbf{Z}^{d, 2}$ and the corresponding mode shape from the polynomial fit are shown in Figs. 13(a) through (d), respectively, based on which observations similar to those associated with noise-free $\mathbf{Z}^{d, 2}$ and $\mathbf{Z}^{u, 2}$ in Fig. 12 can be made, and the damage can also be clearly identified. White noise is then added to $\mathbf{Z}^{d, 2}$ with a signal-to-noise ratio of $60 \mathrm{db}$ to simulate measurement noise. MaximumCDIs, Minimum-CDIs, Mean-CDIs and Gaussian-CDIs associated with $\mathbf{Z}^{d, 2}$ and the corresponding mode shape from the polynomial fit with $s=0.015$ $\mathrm{m}$ in the scheme are shown in Figs. 14(a) through (d), respectively; the existence of the damage is fuzzed in the CDIs due to the measurement noise. Weighted Maximum-CDIs, Minimum-CDIs, Mean-CDIs and Gaussian-CDIs associated with $\mathbf{Z}^{d, 2}$ and the corresponding mode shape from the polynomial fit are shown in Figs. 15(a) through (d), respectively, with $s=0.015 \mathrm{~m}$ in the scheme and $M_{w}=7$ in the weight function. Note that $s=0.015 \mathrm{~m}$ is used here to alleviate adverse effects of the measurement noise on calculating the 
CDIs, while $s=0.002 \mathrm{~m}$ can be used to calculate the CDIs associated with noise-free $\mathbf{Z}^{d, 2}$. While the weight function has been applied, the weighted Gaussian-CDIs cannot be used to identify the damage due to the measurement noise. Similar to the results associated with noise-free $\mathbf{Z}^{d, 2}$ in Fig. 13, the damage can be identified in regions with consistently higher values of the weighted Maximum-CDIs, Minimum-CDIs and Mean-CDIs. While some regions formed by higher values of the CDIs due to the measurement noise can be observed beyond the damage, they do not consistently occur in the weighted CDIs and would not be identified as damage. However, the damage cannot be identified in the weighted Gaussian-CDIs possibly because they are less sensitive to the damage and less robust against measurement noise, compared with the other three weighted CDIs. A possible worse case is that more than one CDI associated with a mode shape are less sensitive to the damage and/or less robust against measurement noise, and one may not identify the damage based on the CDIs. In this case, use of CDIs associated with other mode shapes may be necessary for one to confirm existence of damage, since there does not exist a mode shape that can be used to identify all possible damage and so do not its associated CDIs.

Another finite element model of the damaged plate in Fig. 1(a) is constructed with a dense measurement grid; the model has a total of $240 \times 320$ square plate elements. A mode shape $\mathbf{Z}^{d, 2}$ from the finite element model is presented on a coarse measurement grid with a total of $31 \times 41$ points, as shown in Fig. 10(d). The proper value of $n$ associated with $\mathbf{Z}^{d, 2}$ on the coarse grid is determined to be 8 , and the mode shape from the polynomial fit with $n=8$, denoted by $\mathbf{Z}^{p, 2}$, is shown in Fig. 10(e). Maximum-CDIs, Minimum- 
CDIs, Mean-CDIs and Gaussian-CDIs associated with noise-free $\mathbf{Z}^{d, 2}$ on the coarse grid and the corresponding mode shape from the polynomial fit with $s=0.002 \mathrm{~m}$ in the scheme are shown in Figs. 16(a) through (d), respectively. Similar to the results associated with $\mathbf{Z}^{d, 2}$ on the dense grid in Fig. 13, the damage can also be clearly identified from the CDIs in Fig. 16.

White noise is then added to $\mathbf{Z}^{d, 2}$ on the coarse grid with a signal-to-noise ratio of $60 \mathrm{db}$ to simulate measurement noise. Maximum-CDIs, MinimumCDIs, Mean-CDIs and Gaussian-CDIs associated with the mode shape and the corresponding mode shape from the polynomial fit with $s=0.015 \mathrm{~m}$ in the scheme are shown in Figs. 17(a) through (d), respectively. Similar to Fig. 14, the existence of the damage is fuzzed in the CDIs due to the measurement noise. Associated weighted Maximum-CDIs, Minimum-CDIs, Mean-CDIs and Gaussian-CDIs with $s=0.015 \mathrm{~m}$ in the scheme and $M_{w}=7$ in the weight function are shown in Figs. 18(a) through (d), respectively, where the damage cannot be identified. When white noise is added to $\mathbf{Z}^{d, 2}$ on the coarse grid with an increased signal-to-noise ratio of $75 \mathrm{db}$, the damage still cannot be identified in its Maximum-CDIs, Minimum-CDIs, Mean-CDIs and Gaussian-CDIs with $s=0.015 \mathrm{~m}$ in the scheme, as shown in Figs. 19(a) through (d), respectively. However, the damage can be identified in associated weighted Maximum-CDIs, Minimum-CDIs and Mean-CDIs with $s=0.015 \mathrm{~m}$ in the scheme and $M_{w}=7$ in the weight function, as shown in Figs. 20(a) through (c), respectively, which is similar to the case of $\mathbf{Z}^{d, 2}$ on the dense grid with a signal-to-noise ratio of $60 \mathrm{db}$.

CDIs in Eqs. (14) through (17) consist of differences between CMSs of damaged and undamaged plates. Use of the weight function in Eqs. (28) 
through (31) at a point is equivalent to calculation of weighted sums of the differences within a region centered around the point. The size of the region is determined by the density of a measurement grid and $M_{w}$ in the weight function. The smaller the density, the larger the region; the larger the weight $M_{w}$, the larger the region. Due to the use of the weight function, differences between the CMSs at a point and its neighboring points are attenuated in resulting weighted CDIs. Note that the differences to be attenuated consist of measurement noise and differences between CMSs associated with the corresponding noise-free mode shape of the damaged and undamaged plates. For a mode shape with a certain signal-to-noise ratio and certain $M_{w}$, the smaller the density of the measurement grid, the larger the differences to be attenuated, and damage may become unidentifiable in resulting weighted CDIs when the differences are too large; it can be verified by comparing Figs. 14 and 18, where the signal-to-noise ratios of $\mathbf{Z}^{d, 2}$ are $60 \mathrm{db}$ and $M_{w}=7$. Since the density of the measurement grid in the former is large enough, the damage can be identified, but it cannot be in the latter due to the relatively small density of the measurement grid. For a mode shape on a measurement grid with a certain density and certain $M_{w}$, the higher the signal-to-noise ratio of the mode shape, the smaller the differences to be attenuated, and damage might become identifiable in resulting weighted CDIs when the differences are small; it can be verified by comparing Figs. 18 and 20, where densities of the measurement grids are the same and $M_{w}=7$. Since the signal-to-noise ratio of $\mathbf{Z}^{d, 2}$ in the latter is high enough, the damage can be identified, but it cannot be in the former due to the low signal-to-noise ratio of $\mathbf{Z}^{d, 2}$. 
The proposed damage identification method is applicable and robust to mode shapes associated with low and high elastic modes on dense and coarse measurement grids. One can infer that use of a coarse measurement grid can lead to a lower spatial resolution in resulting CDIs and the smallest size of identifiable damage would be larger. For a mode shape with relatively large measurement noise, i.e., a mode shape with a relatively low signal-to-noise ratio, use of a dense measurement grid is recommended so that effects of the measurement noise can be alleviated and the existence of damage can be manifested in weighted CDIs. In practice, one can increase the signal-tonoise ratio of a mode shape by increasing accuracy of measurement and/or increasing the level of excitation, and damage can still be identifiable even when a relatively coarse measurement grid is used in this case. The method can be applied to some real structures with curved surfaces, such as wind turbine blades.

\section{Experimental Investigation}

\subsection{Experimental setup}

A damaged aluminum plate that had a length of $500.00 \mathrm{~mm}$, a width of $400.00 \mathrm{~mm}$ and a thickness of $4.75 \mathrm{~mm}$ was constructed; its dimensions are shown in Fig. 21(a). The plate was hung using two nylon cords to simulate free boundary conditions, as shown in Fig. 21(b). The damage was a machined thickness reduction area, which had a length of $40.00 \mathrm{~mm}$, a width of $40.00 \mathrm{~mm}$ and a depth of $0.5 \mathrm{~mm}$; the depth was about $10 \%$ of the thickness of the undamaged portion of the plate, as shown in Fig. 21(c). In order to validate the simulated free boundary conditions of the 
plate, an experimental modal analysis was performed on it, where a PCB 086-D80 miniature impact hammer and a Polytec PSV-500 scanning laser Doppler vibrometer system were used to excite the plate and measure its response (Fig. 21(b)), respectively. In the experimental modal analysis, a fixed excitation point was impacted using the hammer, and the response of a measurement point was measured to yield five FRFs, which were averaged and analyzed using PolyMax of LMS Test.Lab Rev.9b to obtain natural frequencies of the plate. The lowest measured elastic natural frequency of the plate was $76.5 \mathrm{~Hz}$. Since the highest measured natural frequency of rigid body modes of the plate in the setup was $2.1 \mathrm{~Hz}$, which was much smaller than $10 \%$ of the lowest measured elastic natural frequency, the free boundary conditions were validated [23]. A rectangular measurement area of the opposite surface of the plate to the damaged one was used for mode shape measurement; it had a length of $395.9 \mathrm{~mm}$ and a width of $488.1 \mathrm{~mm}$, as shown in Fig. 21(d). The rectangular area is slightly smaller than that of the plate in that there were two holes drilled on two top corners of the plate for hanging and clearances between edges of the plate and the rectangular area were reserved to prevent the laser of the scanning laser Doppler vibrometer system from reaching the two holes. The measurement area was sprayed with spot checker to enhance laser reflection that directly determined signal-tonoise ratios of laser measurements.

In order to accurately measure mode shapes of the damaged plate without incurring unwanted mass loading, non-contact excitation and measurements were performed [26], as shown in Figs. 21(b) and (e): an electric speaker with a wood box faced the damaged surface of the plate and generated acoustic 
excitation onto it, and the scanning laser Doppler vibrometer system measured velocities of measurement points in the measurement area. There were totally $115 \times 177$ measurement points in a measurement grid on the area, whose elements were rectangular and had a length of $3.44 \mathrm{~mm}$ and a width of $2.76 \mathrm{~mm}$, as shown in Fig. 21(d). Acoustic excitation in the form of a sine wave with a constant magnitude and frequency was used to excite the plate, and the velocities of the measurement points were measured using the "FastScan" mode of the scanning laser Doppler vibrometer system. In the "FastScan" mode, a laser spot from the scanning laser Doppler vibrometer system stays at a measurement point on a structure to measure its response for a user-defined number of periods of the sine wave and then moves to the next measurement point. The duration for the laser spot to stay at one measurement point depends on the number of periods of the sine wave and sampling frequency of the system, and it can be as short as a ten-thousandth of a second. Due to this feature, a steady-state vibration shape of the structure under sinusoidal excitation can be measured in a point-by-point, but automatic and rapid, manner. In this measurement, the excitation signal given to the speaker was used for a reference signal, with which a vibration shape with a correct phase at each measurement point can be obtained [27]. The mode shape of the plate at the natural frequency of $1349 \mathrm{~Hz}$, denoted by $\mathbf{Z}^{\text {exp }}$, was measured with an excitation frequency of $1349 \mathrm{~Hz}$ to experimentally investigate the effectiveness of the proposed method; the duration for the laser spot to stay at each measurement point was about $0.06 \mathrm{~s}$. In order to transform the current measurement grid to one with square elements and increase spatial resolution of CDIs, $\mathbf{Z}^{\exp }$ was interpolated using the Matlab 
function "griddata" with the option "natural" on a measurement grid with $199 \times 245$ measurement points, as shown in Fig. 22(a), which has square elements with a side length of $1.99 \mathrm{~mm}$. For simplicity, the interpolated $\mathbf{Z}^{\exp }$ is denoted by $\mathbf{Z}^{\exp }$ herein.

\section{2. $C M S$ and damage identification results}

Maximum, minimum, mean and Gaussian CMSs associated with $\mathbf{Z}^{\text {exp }}$ from the scheme with $s=0.002 \mathrm{~m}, s=0.005 \mathrm{~m}$ and $s=0.015 \mathrm{~m}$ are shown in Figs. 23 through 25, respectively. In Fig. 23, global trends of the four CMSs associated with $\mathbf{Z}^{\exp }$ from the scheme with $s=0.002 \mathrm{~m}$ could be observed with severe noise caused by measurement noise. When $s=0.005$ m, the scheme yielded CMSs with much lower noise levels and their global trends became clearer than those from the scheme with $s=0.002 \mathrm{~m}$, as shown in Fig. 24. When $s=0.015 \mathrm{~m}$, associated CMSs were of the best qualities compared with those from the scheme with $s=0.002 \mathrm{~m}$ and 0.005 m. Adverse effects of measurement noise were mostly eliminated with a larger value of $s$.

To determine the proper order of a polynomial to fit $\mathbf{Z}^{\exp }$, fit associated with $\mathbf{Z}^{\exp }$ for different $n$ were calculated, as shown in Fig. 26(a). Similar to the numerical example in Sec. 2.4, fit converged to a certain value as $n$ increased, as shown in Fig. 26(b). Associated con for different $n$ were calculated, as shown in Fig. 26(c). The proper order of the polynomial to fit $\mathbf{Z}^{\exp }$ was determined to be 15 , since $\operatorname{con}=0.35 \%$, which was lower than the prescribed threshold value for con, as shown in Fig. 26(d). The mode shape from the polynomial fit with $n=15$ is shown in Fig. 22(b), and the MAC value associated with the mode shape from the polynomial fit and $\mathbf{Z}^{\exp }$ was 
$99.99 \%$.

Maximum-CDIs, Minimum-CDIs, Mean-CDIs and Gaussian-CDIs associated with $\mathbf{Z}^{\exp }$ and the corresponding mode shape from the polynomial fit with $s=0.002 \mathrm{~m}, 0.005 \mathrm{~m}$ and $0.015 \mathrm{~m}$ in the scheme are shown in Figs. 27 through 29, respectively. In Fig. 27, the damage could not be identified from the four CDIs. The reason was that the associated CMSs were noisy since effects of measurement noise were amplified, and those of the damage on the CMSs were fussed. When $s$ increased to $0.005 \mathrm{~m}$ in the scheme, ridges and regions that were formed by higher values of the CDIs in the neighborhood of and beyond the damage could be observed, as shown in Fig. 28. However, one could not identify the damage based on the CDIs due to the ridges and regions beyond the damage. When $s=0.015 \mathrm{~m}$, ridges and regions similar to those in Fig. 28(a), (b) and (c) could still be observed in the Maximum-CDIs, Minimum-CDIs and Mean-CDIs, as shown in Fig. 29(a), (b) and (c), respectively. The difference is that higher values of the CDIs could be observed in the neighborhood of the damage when $s=0.015 \mathrm{~m}$. In 29(d), higher values of the CDIs could be observed in the neighborhood of the damage; some higher values of the CDIs could also be observed in some regions beyond the damage. By use of the four CDIs in Fig. 29, one can identify the damage in regions with consistently higher values of the CDIs.

To alleviate adverse effects of measurement noise on the CDIs, the weight function in Eq. (27) with $M_{w}=7$ was applied to the CDIs in Figs. 27 through 29, based on Eqs. (28) through (31), and resulting weighted CDIs are shown in Figs. 30 through 32, respectively. When $s=0.002 \mathrm{~m}$, relatively high values could be observed in the weighted Maximum-CDIs, Minimum- 
CDIs and Gaussian-CDIs in the neighborhood of and beyond the damage, and one could not identify the damage based on the three CDIs; higher values of the weighted Mean-CDIs could be identified in the neighborhood of the damage. When $s=0.005 \mathrm{~m}$ and $0.015 \mathrm{~m}$, one could clearly identify the damage in neighborhoods of consistently higher values of weighted CDIs, as shown in Figs. 31 and 32, respectively. CDIs in Fig. 32 were less noisy than those in Fig. 31; consistently higher values of CDIs were mainly in the neighborhood of the damage in Fig. 32. Comparing CDIs with the same value of $s$ in the scheme, one could see that use of the weight function could denoise the CDIs and manifest the damage with a smaller value of $s$.

A finite element model of the damaged plate was constructed, and a noise-free undamped mode shape associated with the same mode as that of $\mathbf{Z}^{\text {exp }}$ was calculated and denoted by $\mathbf{Z}^{\text {num }}$, as shown in Fig. 22(c). MaximumCDIs, Minimum-CDIs, Mean-CDIs and Gaussian-CDIs associated with $\mathbf{Z}^{\text {num }}$ and the corresponding mode shape from the polynomial fit with $s=0.015 \mathrm{~m}$ in the scheme are shown in Figs. 33(a) through (d), respectively; weighted Maximum-CDIs, Minimum-CDIs, Mean-CDIs and Gaussian-CDIs associated with $\mathbf{Z}^{\text {num }}$ and the corresponding mode shape from the polynomial fit with $s=0.015 \mathrm{~m}$ in the scheme and $M_{w}=7$ in the weight function are shown in Figs. 34(a) through (d), respectively. Similar to the experimental damage identification results in Figs. 29 and 32, consistently higher values of the CDIs and weighted CDIs associated with $\mathbf{Z}^{\text {num }}$ occur in the neighborhood of the damage, and they resemble those associated with $\mathbf{Z}^{\text {exp }}$, which verifies the experimental damage identification results. The CDIs associated with $\mathbf{Z}^{\text {num }}$ and the corresponding mode shape from the polynomial fit resemble the 
associated weighted CDIs, as shown in Figs. 33 and 34, respectively, which indicates that use of the weight function does not affect damage identification results when a mode shape is noise-free. Effectiveness of using the weight function in alleviating adverse effects of measurement noise on the CDIs and manifesting existence of damage in the CDIs can be verified by comparing the CDIs associated with $\mathbf{Z}^{\text {num }}$ in Fig. 33 with the weighted CDIs associated with $\mathbf{Z}^{\text {exp }}$ in Fig. 32, since the latter, which have lower noise levels than the CDIs associated with $\mathbf{Z}^{\text {exp }}$ in Fig. 29, resemble the former.

\section{Conclusions}

A new non-model-based plate damage identification method based on principal, mean and Gaussian CMSs is proposed. It can be applied to a damaged plate without use of any a priori information of the associated undamaged one, if the undamaged plate is geometrically smooth and made of materials that have no stiffness and mass discontinuities. A multi-scale differential-geometry scheme is proposed to calculate the CMSs associated with a mode shape. The advantage of the scheme is that adverse effects of measurement noise could be alleviated with use of a larger value of the scale parameter. Differences between the aforementioned CMSs of a damaged plate and those associated with a mode shape from a polynomial that fits the corresponding mode shape of the damaged plate are processed to yield four CDIs. Based on the fitting index, a mode shape from a polynomial that fits the corresponding mode shape of a damaged plate would have a higher level of approximation as the order of the polynomial increases. A threshold value is used for the convergence index, based on which the order of the polynomial 
can be properly determined. CMSs associated with a mode shape from a polynomial of a properly determined order can be used to eliminate global trends of CMSs associated with the corresponding mode shape of a damaged plate. Use of a weight function can alleviate effects of measurement noise on the CDIs and shorten the process of progressively testing different values of the scale parameter. The proposed method is applicable and robust to mode shapes associated with low and high elastic modes; it is recommended that one increase the signal-to-noise ratio of a mode shape and use a dense measurement grid to alleviate effects of measurement noise. The proposed method was experimentally applied to a plate with damage in the form of a machined thickness reduction area. The damage was successfully identified by locating consistently higher values of the CDIs. A larger value of the scale parameter in the scheme can manifest existence of damage in the CDIs; use of the weight function can manifest existence of damage even with a small value of the scale parameter in the scheme. The experimental damage identification results were numerically verified by applying the proposed method to the mode shape associated with the same mode as that of the measured mode shape from a finite element model of the damaged plate.

\section{Acknowledgement}

The authors are grateful for the financial support from the National Science Foundation under Grant Numbers CMMI-1229532 and CMMI-1335024. 


\section{References}

[1] K. He, W. D. Zhu, Detection of damage and loosening of bolted connections in structures using changes in natural frequencies, ASNT Materials Evaluation 68 (6) (2010) 721-732.

[2] K. He, W. D. Zhu, vibration-based structural damage detection method and its applications to engineering structures, International Journal of Smart and Nano Materials 2 (3) (2011) 194-218.

[3] W. D. Zhu, K. He, Detection of damage in space frame structures with l-shaped beams and bolted joints using changes in natural frequencies, Journal of Vibration and Acoustics 135 (5) (2013) 051001.

[4] K. He, W. D. Zhu, Detecting loosening of bolted connections in a pipeline using changes in natural frequencies, Journal of Vibration and Acoustics 136 (3) (2014) 034503.

[5] C. P. Ratcliffe, A frequency and curvature based experimental method for locating damage in structures, Journal of vibration and acoustics 122 (3) (2000) 324-329.

[6] M. K. Yoon, D. Heider, J. W. Gillespie, C. P. Ratcliffe, R. M. Crane, Local damage detection using the two-dimensional gapped smoothing method, Journal of Sound and Vibration 279 (1) (2005) 119-139.

[7] Y. Zhang, L. Wang, S. T. Lie, Z. Xiang, Damage detection in plates structures based on frequency shift surface curvature, Journal of Sound and Vibration 332 (25) (2013) 6665-6684. 
[8] W. Xu, M. Cao, W. Ostachowicz, M. Radzieński, N. Xia, Twodimensional curvature mode shape method based on wavelets and teager energy for damage detection in plates, Journal of Sound and Vibration 347 (2015) 266-278.

[9] P. Qiao, K. Lu, W. Lestari, J. Wang, Curvature mode shape-based damage detection in composite laminated plates, Composite Structures 80 (3) (2007) 409-428.

[10] D. Wu, S. S. Law, Damage localization in plate structures from uniform load surface curvature, Journal of Sound and Vibration 276 (1) (2004) $227-244$.

[11] G. Teza, A. Galgaro, F. Moro, Contactless recognition of concrete surface damage from laser scanning and curvature computation, NDT \& E International 42 (4) (2009) 240-249.

[12] C.-C. Chang, L.-W. Chen, Damage detection of a rectangular plate by spatial wavelet based approach, Applied Acoustics 65 (8) (2004) 819832.

[13] M. Rucka, K. Wilde, Application of continuous wavelet transform in vibration based damage detection method for beams and plates, Journal of Sound and Vibration 297 (3) (2006) 536-550.

[14] E. Douka, S. Loutridis, A. Trochidis, Crack identification in plates using wavelet analysis, Journal of sound and vibration 270 (1) (2004) 279-295.

[15] P. Cornwell, S. W. Doebling, C. R. Farrar, Application of the strain 
energy damage detection method to plate-like structures, Journal of Sound and Vibration 224 (2) (1999) 359-374.

[16] C. Surace, R. Saxena, M. Gherlone, H. Darwich, Damage localisation in plate like-structures using the two-dimensional polynomial annihilation edge detection method, Journal of Sound and Vibration 333 (21) (2014) $5412-5426$.

[17] C. Surace, R. Archibald, R. Saxena, On the use of the polynomial annihilation edge detection for locating cracks in beam-like structures, Computers \& Structures 114 (2013) 72-83.

[18] B. O'neill, Elementary differential geometry, 2nd Edition, Academic press, Oxford, UK, 2006.

[19] S. Timoshenko, S. Woinowsky-Krieger, Theory of Plates and Shells, 2nd Edition, McGraw-hill New York, 1959.

[20] M. Meyer, M. Desbrun, P. Schröder, A. H. Barr, Discrete differentialgeometry operators for triangulated 2-manifolds, in: Visualization and mathematics III, Springer, 2003, pp. 35-57.

[21] Y. F. Xu, W. D. Zhu, J. Liu, Y. M. Shao, Identification of embedded horizontal cracks in beams using measured mode shapes, Journal of Sound and Vibration 333 (23) (2014) 6273-6294.

[22] A. K. Pandey, M. Biswas, M. M. Samman, Damage detection from changes in curvature mode shapes, Journal of Sound and Vibration 145 (2) (1991) 321-332. 
[23] D. J. Ewins, Modal Testing: Theory, Practice and Application, 2nd Edition, Research Studies Press, Hertfordshire, UK, 2000.

[24] J. Nocedal, S. Wright, Numerical optimization, Springer Science \& Business Media, New York, USA, 2006.

[25] I. Cox, M. Gaudard, Discovering Partial Least Squares with JMP, SAS Institute, Maryland, USA, 2013.

[26] Y. F. Xu, W. D. Zhu, Operational modal analysis of a rectangular plate using non-contact excitation and measurement, Journal of Sound and Vibration 332 (20) (2013) 4927-4939.

[27] B. Schwarz, S. Richardson, M. Richardson, Using mode shapes for real time ods animation, in: Topics in Modal Analysis, Volume 10, Springer, 2015, pp. 103-113. 


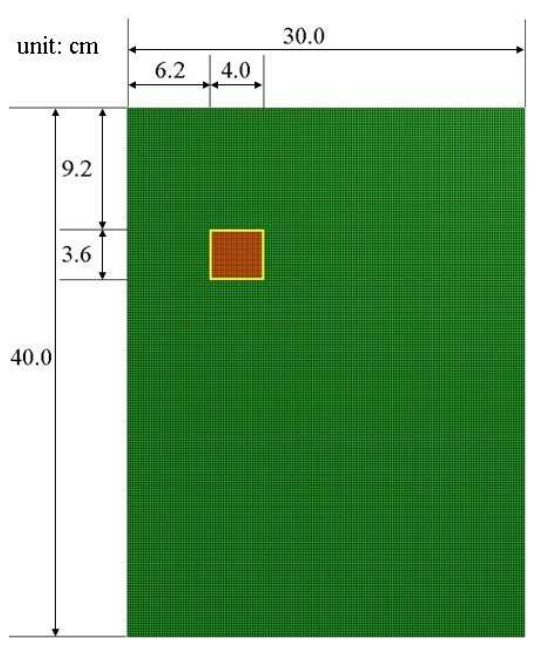

(a)

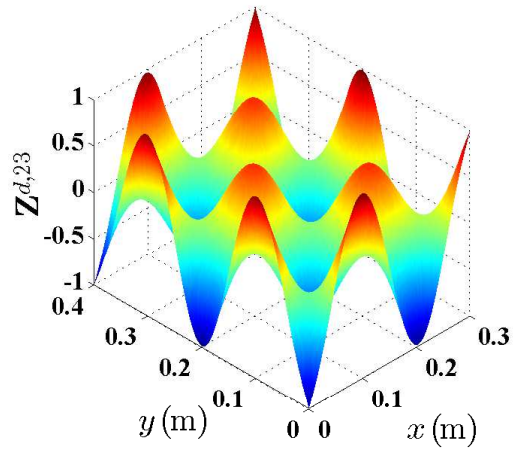

(b)

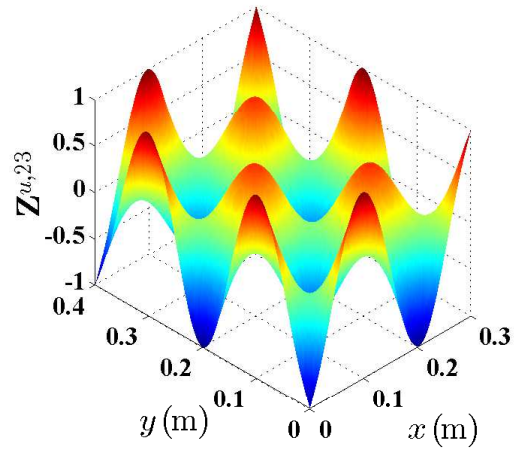

(c)

Fig. 1 (a) Finite element model of a plate with damage in the form of a thickness reduction area, (b) the 23-rd mode shape of the damaged plate and (c) the 23-rd mode shape of an undamaged plate of the same dimensions and material properties as the damaged one. 


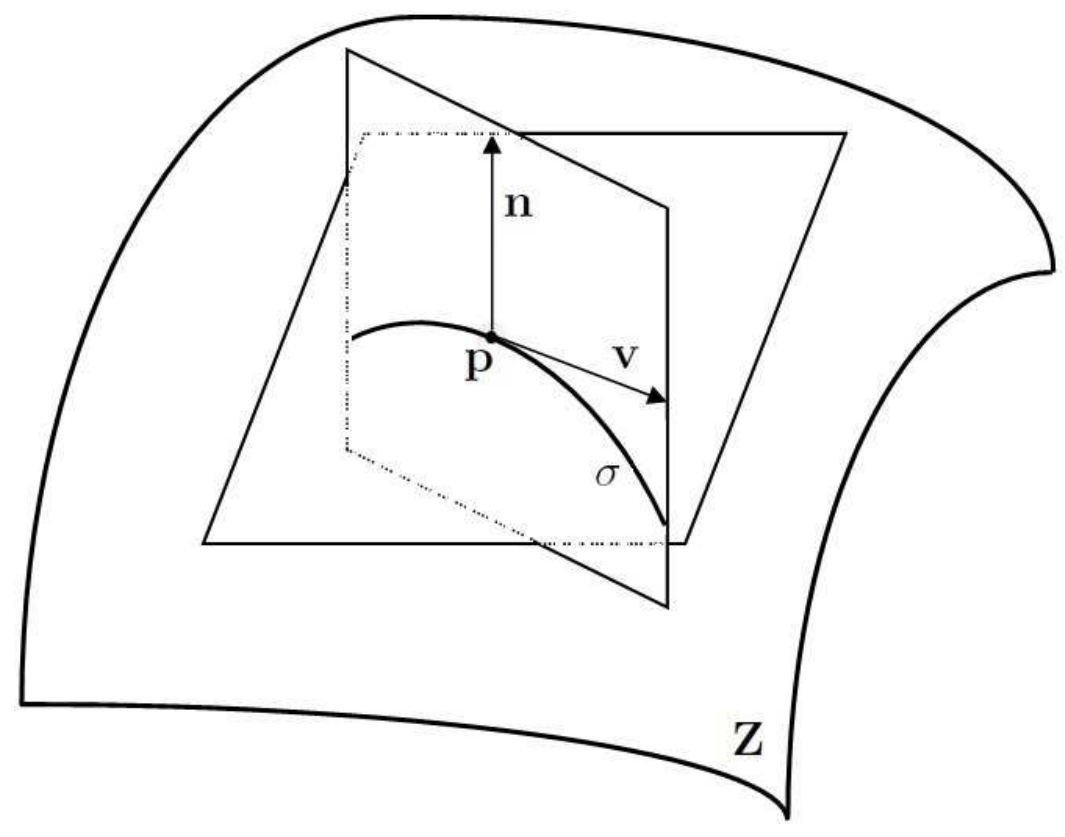

Fig. 2 Point $\mathbf{p}$ on a mode shape $\mathbf{Z}$, a unit normal vector $\mathbf{n}$ associated with $\mathbf{p}$, a unit tangent vector $\mathbf{v}$ associated with $\mathbf{p}$, and a curve $\sigma$ obtained by intersecting $\mathbf{Z}$ with the plane determined by $\mathbf{n}$ and $\mathbf{v}$. 


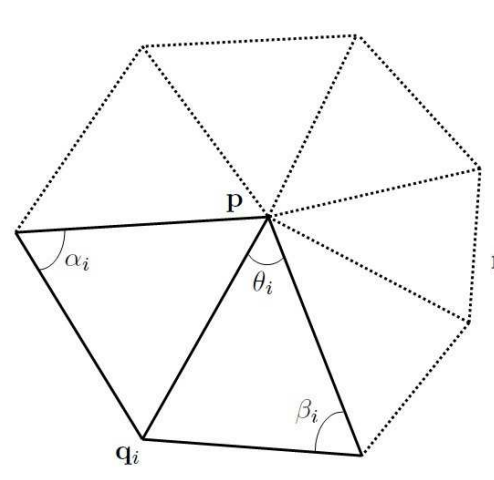

(a)

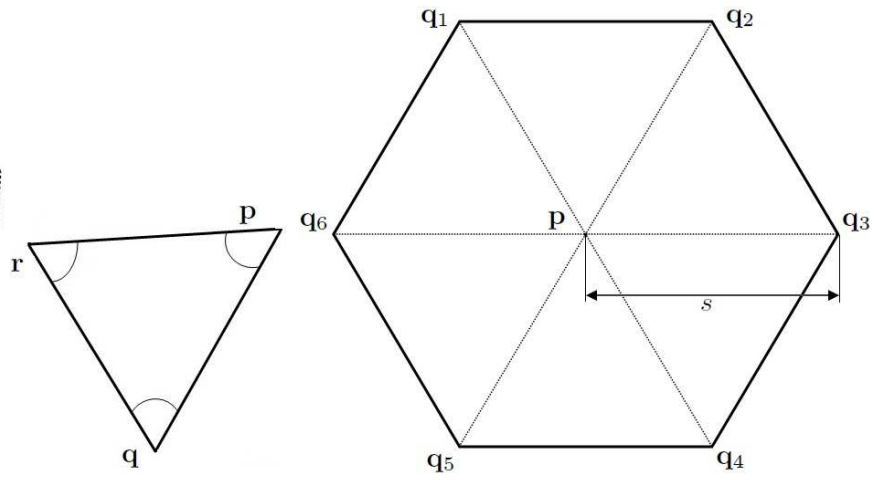

(b)

(c)

Fig. 3 (a) One-ring neighborhood of a triangulated mesh associated with $\mathbf{p}$, (b) an acute triangle $\triangle \mathbf{p q r}$ and (c) a hexagonal one-ring neighborhood with a side length of $s$. 
$A_{\mathbf{P}}=0$

For each triangle in the one-ring neighborhood of $\mathbf{p}$

If the triangle is acute,

$$
A_{\mathbf{P}}=A_{\mathbf{P}}+A_{\text {Voronoi }}
$$

Else

If $\angle \mathbf{p}$ is obtuse

$$
A_{\mathbf{P}}=A_{\mathbf{P}}+\frac{A}{2}
$$

Else

$$
A_{\mathbf{P}}=A_{\mathbf{P}}+\frac{A}{4}
$$

Fig. 4 Pseudo-code for calculating the new surface area $A_{\mathbf{p}}$ associated with $\mathbf{p}$. 


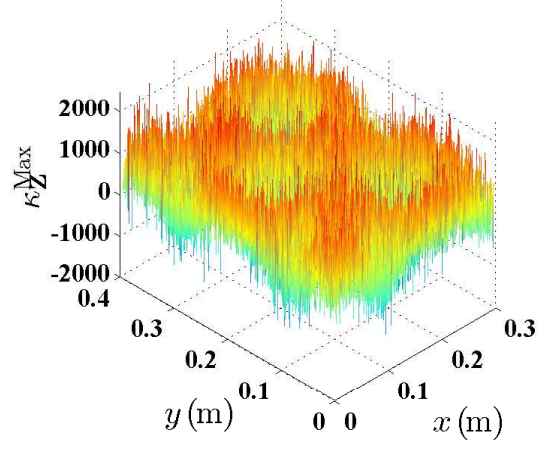

(a)

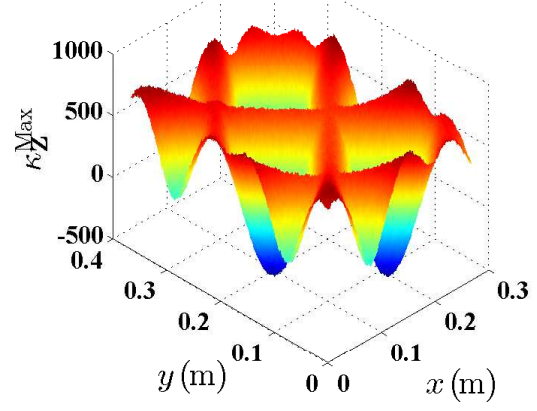

(c)

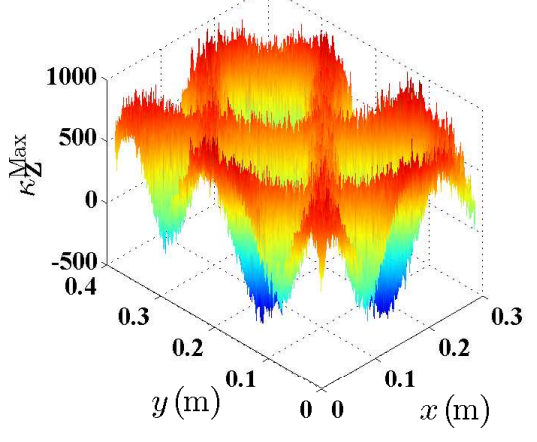

(b)

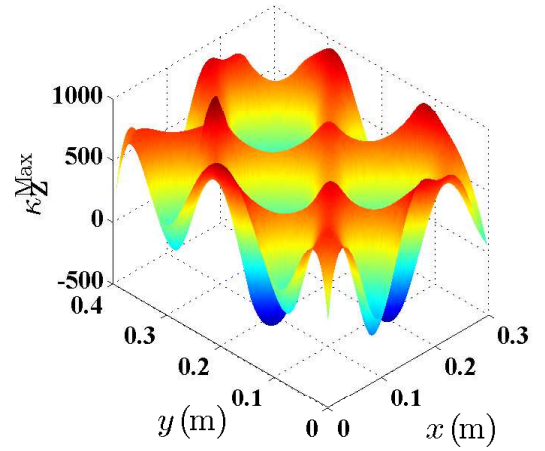

(d)

Fig. 5 Maximum CMSs associated with $\mathbf{Z}^{d, 23}$ with measurement noise from the scheme with (a) $s=0.002 \mathrm{~m}$, (b) $s=0.005 \mathrm{~m}$ and (c) $s=0.015 \mathrm{~m}$; (d) the maximum CMS associated with noise-free $\mathbf{Z}^{d, 23}$ from the scheme with $s=0.002$ $\mathrm{m}$. 


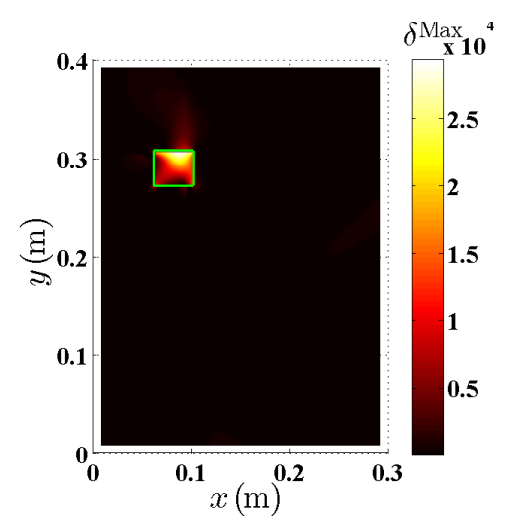

(a)

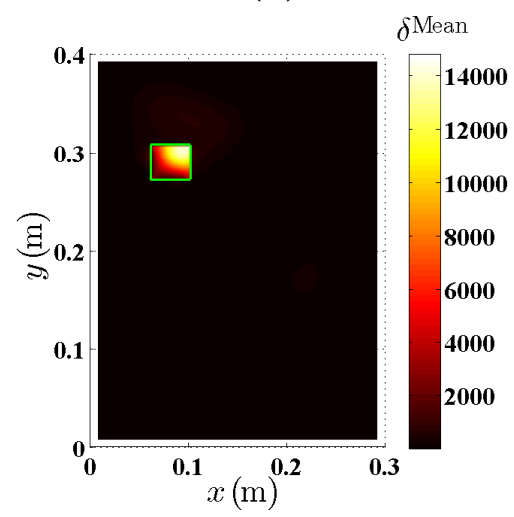

(c)

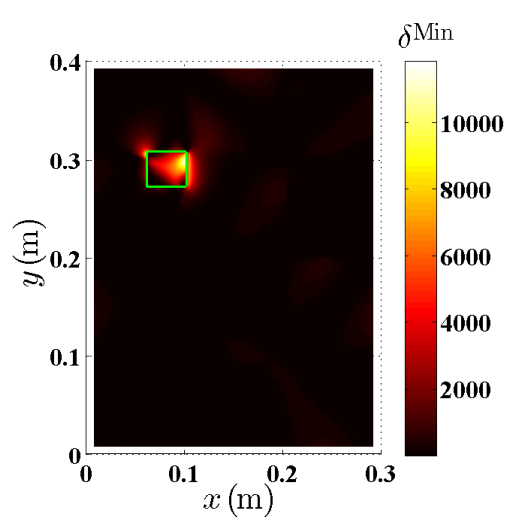

(b)

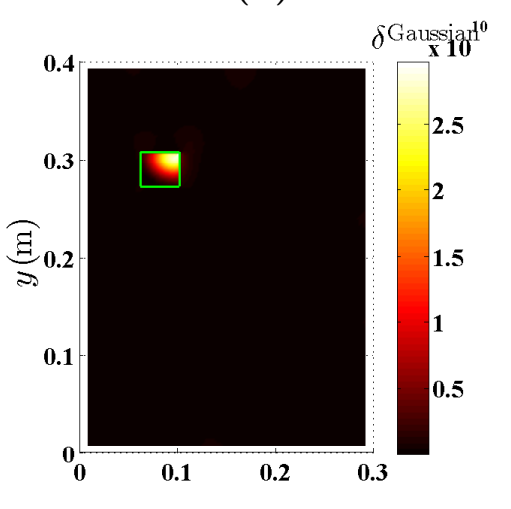

(d)

Fig. 6 (a) Maximum-CDIs, (b) Minimum-CDIs, (c) Mean-CDIs and (d) GaussianCDIs associated with noise-free $\mathbf{Z}^{d, 23}$ and $\mathbf{Z}^{u, 23} ; s=0.002 \mathrm{~m}$ in the scheme. 
(a)

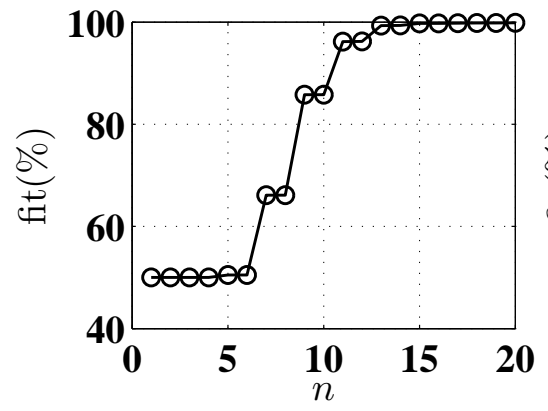

(c)

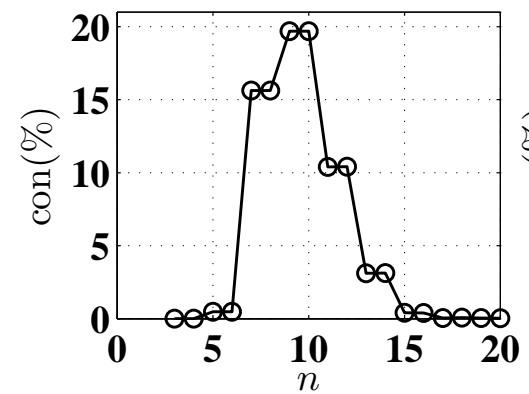

(b)

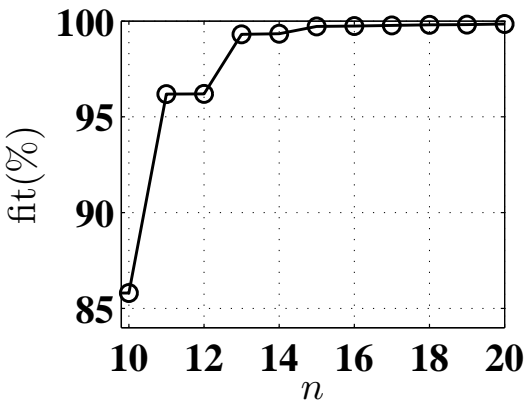

(d)

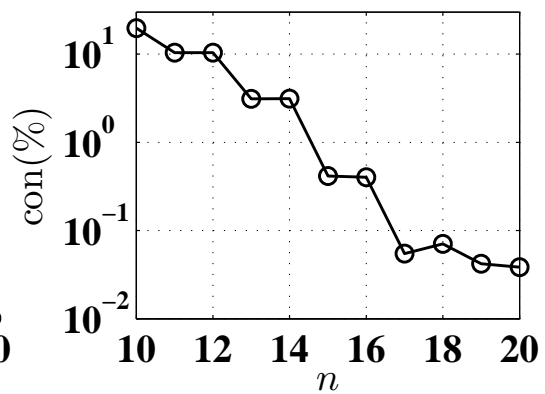

Fig. 7 (a) Fitting indices fit associated with $\mathbf{Z}^{d, 23}$ for different $n$, (b) an enlarged view of fit, (c) the convergence indices con associated with $\mathbf{Z}^{d, 23}$ for different $n$ and (d) an enlarged view of con. 


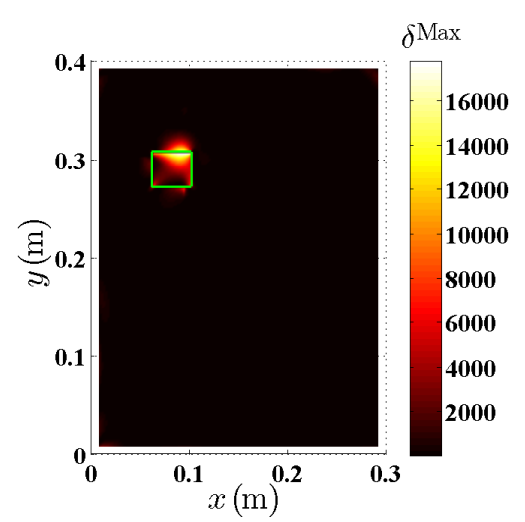

(a)

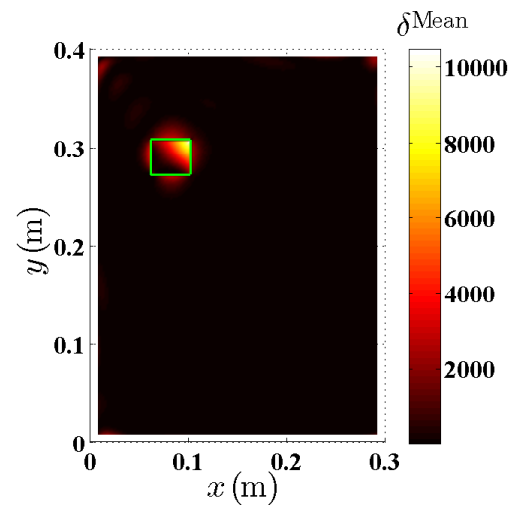

(c)

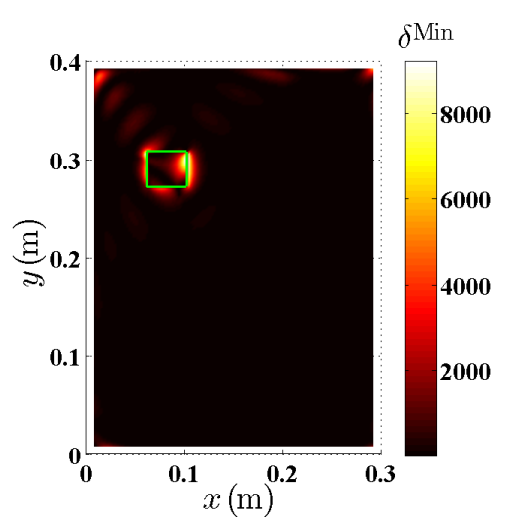

(b)

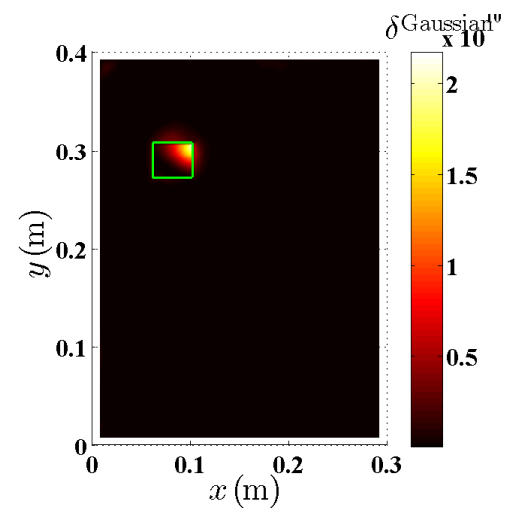

(d)

Fig. 8 (a) Maximum-CDIs, (b) Minimum-CDIs, (c) Mean-CDIs and (d) GaussianCDIs associated with noise-free $\mathbf{Z}^{d, 23}$ and the corresponding mode shape from the polynomial fit with $n=15 ; s=0.002 \mathrm{~m}$ in the scheme. 


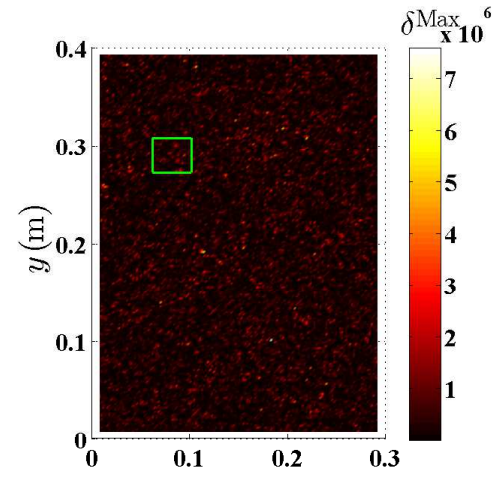

(a)

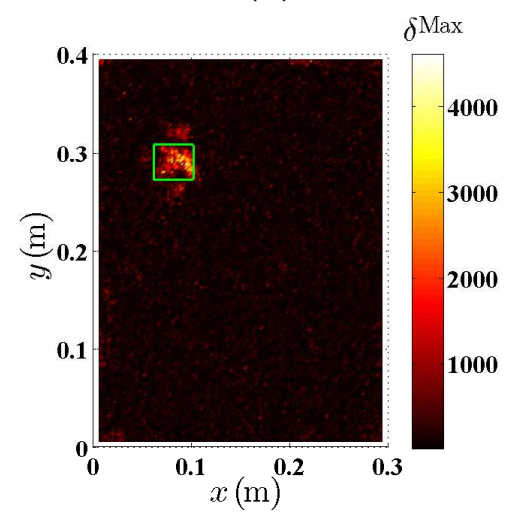

(c)

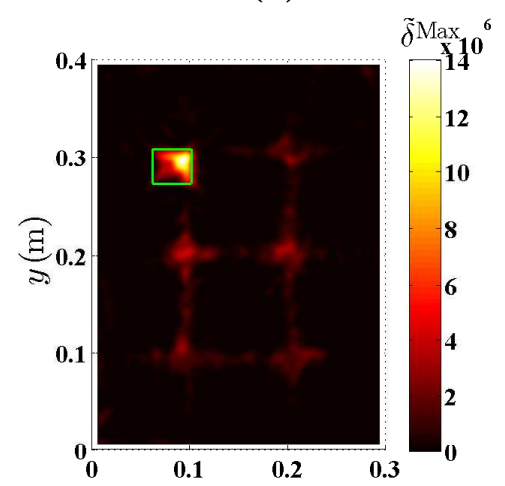

(e)

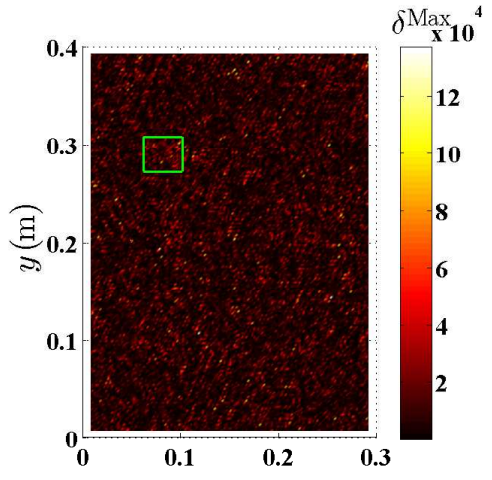

(b)

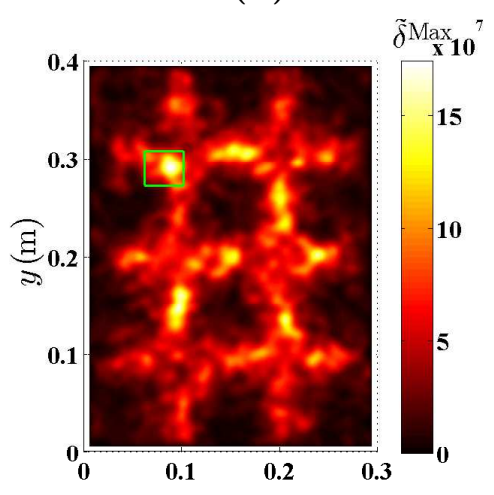

(d)

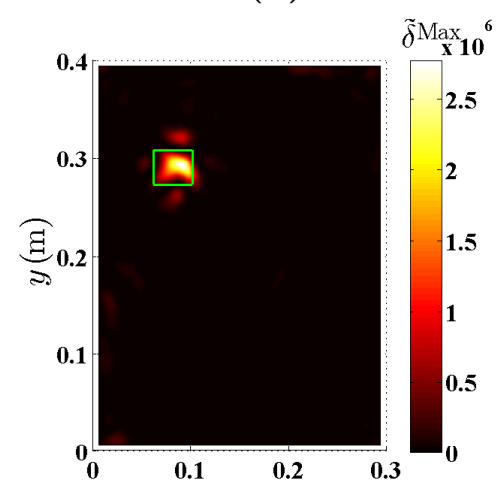

(f)

Fig. 9 Maximum-CDIs associated with $\mathbf{Z}^{d, 23}$ and the corresponding mode shape from the polynomial fit with (a) $s=0.002 \mathrm{~m}$, (b) $s=0.005 \mathrm{~m}$ and (c) $s=$ $0.015 \mathrm{~m}$ in the scheme; weighted Maxiqygum-CDIs associated with $\mathbf{Z}^{d, 23}$ and the corresponding mode shape from the polynomial fit with (d) $s=0.002 \mathrm{~m}$, (e) $s=0.005 \mathrm{~m}$ and (f) $s=0.015 \mathrm{~m}$ in the scheme. The order of the polynomial fit is $n=15$, and the scale of the weight function is $M_{w}=7$. 


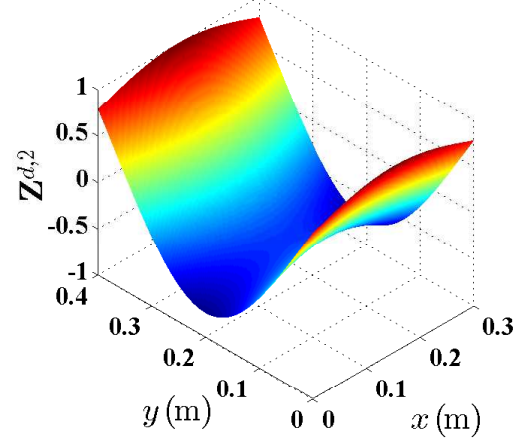

(a)

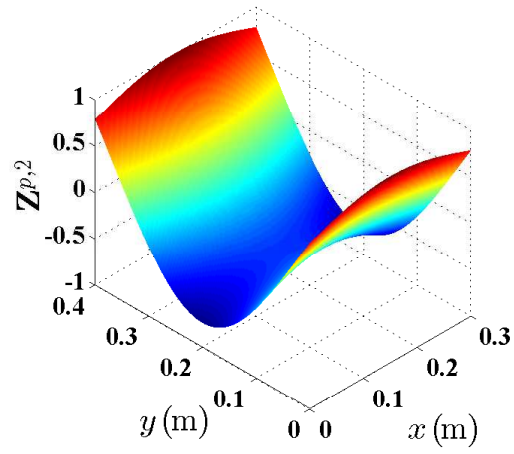

(c)

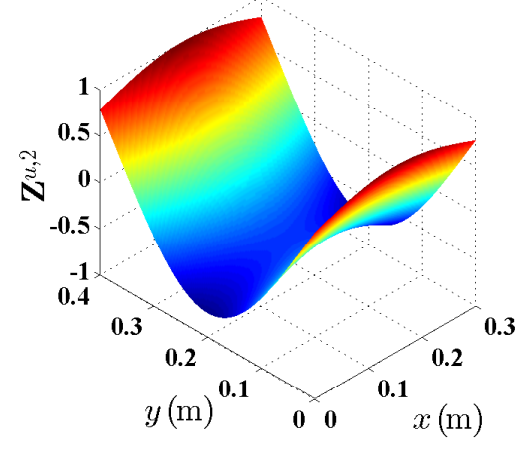

(b)

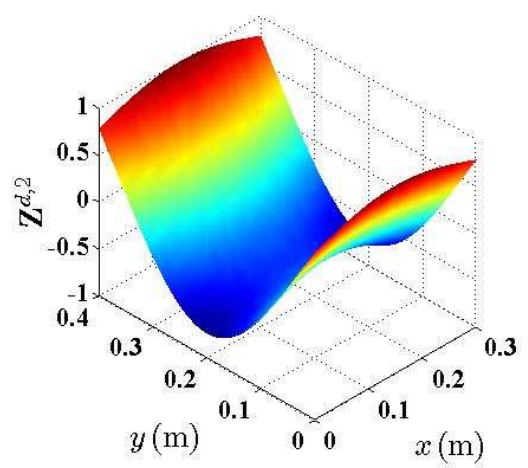

(d)

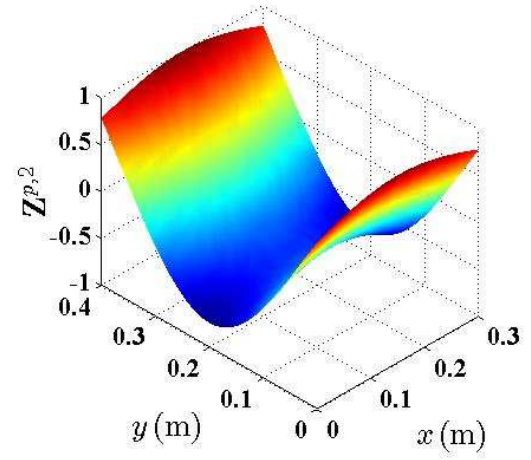

(e)

Fig. 10 (a) Mode shape $\mathbf{Z}^{d, 2}$ of the damaged plate, (b) $\mathbf{Z}^{u, 2}$ of the undamaged plate, (c) $\mathbf{Z}^{p, 2}$ from a polynomial that fits $\mathbf{Z}^{d, 2}$ in (a) with $n=8$, (d) $\mathbf{Z}^{u, 2}$ of the damaged plate on a coarse measurement 1 rid and (e) $\mathbf{Z}^{p, 2}$ from a polynomial that fits $\mathbf{Z}^{d, 2}$ in (d) with $n=8$. 
(a)

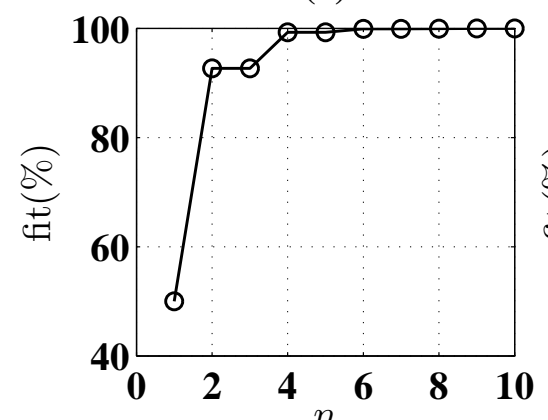

(c)

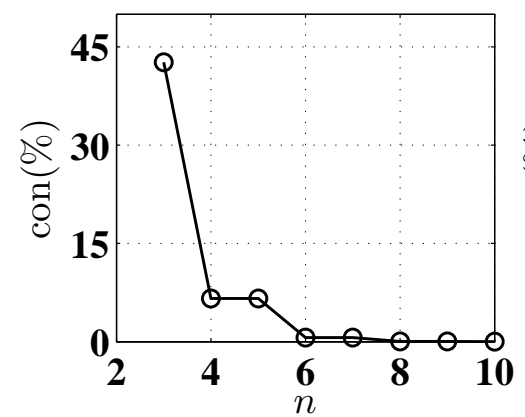

(b)

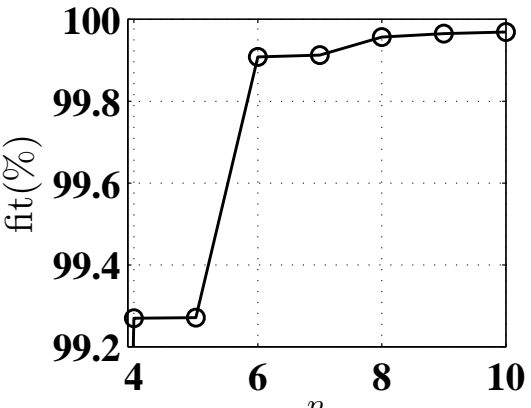

(d)

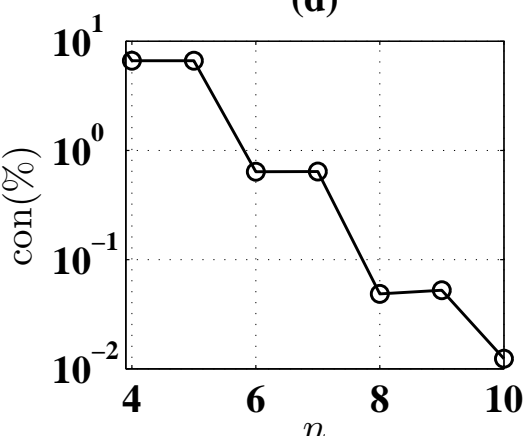

Fig. 11 (a) Fitting indices fit associated with $\mathbf{Z}^{d, 2}$ for different $n$, (b) an enlarged view of fit, (c) the convergence indices con associated with $\mathbf{Z}^{d, 2}$ for different $n$ and (d) an enlarged view of con. 


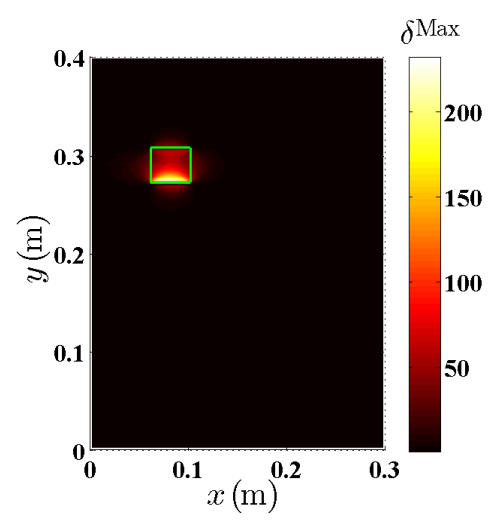

(a)

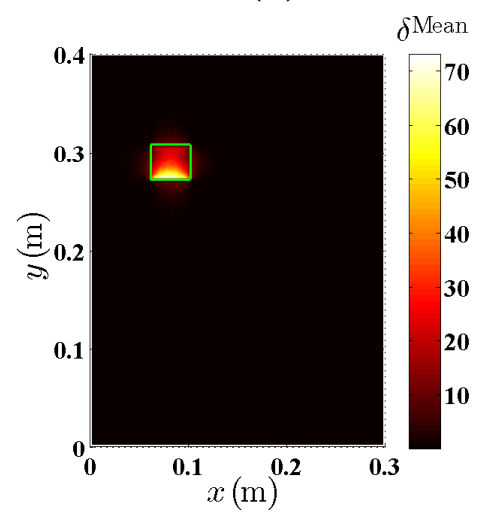

(c)

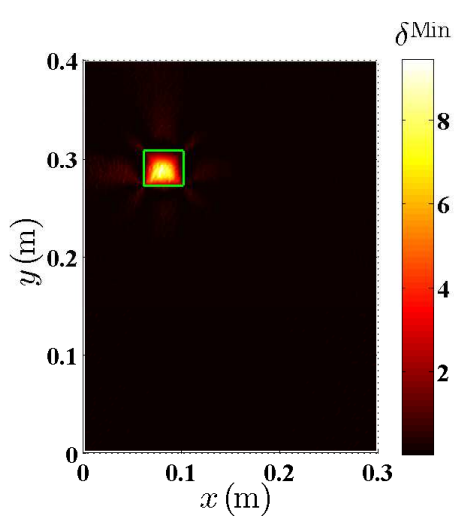

(b)

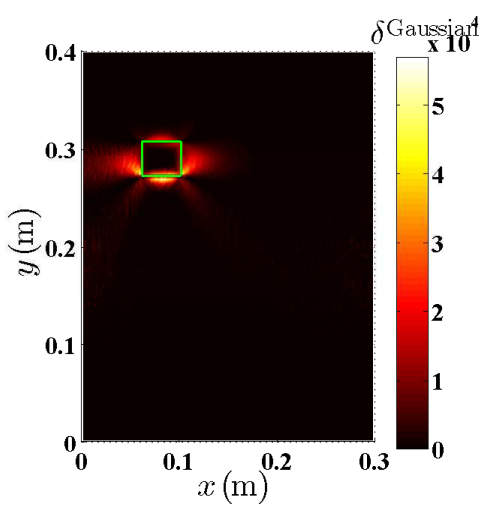

(d)

Fig. 12 (a) Maximum-CDIs, (b) Minimum-CDIs, (c) Mean-CDIs and (d) Gaussian-CDIs associated with noise-free $\mathbf{Z}^{d, 2}$ and $\mathbf{Z}^{u, 2} ; s=0.002 \mathrm{~m}$ in the scheme. 


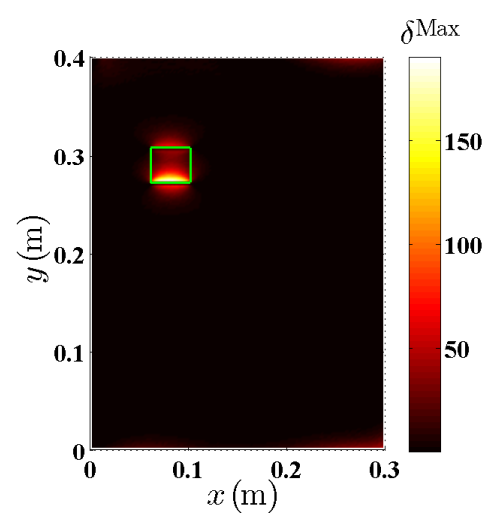

(a)

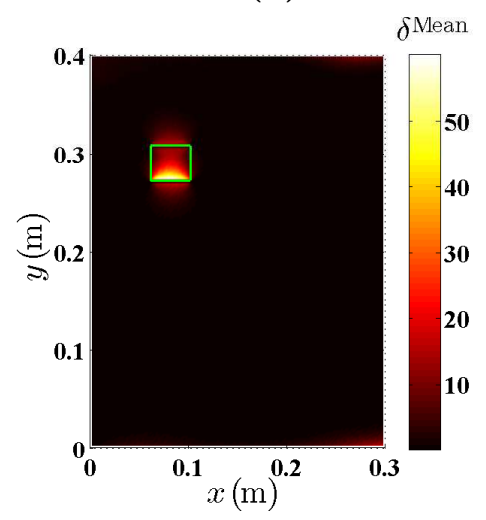

(c)

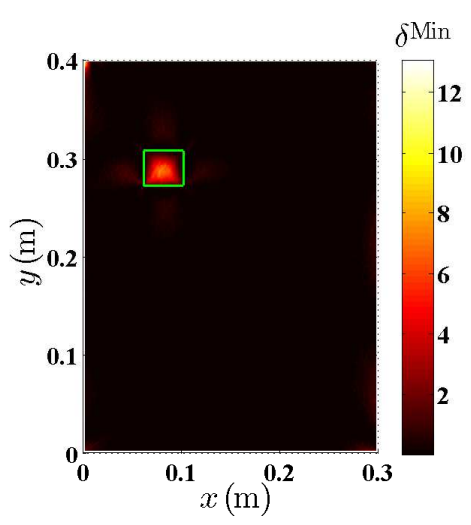

(b)

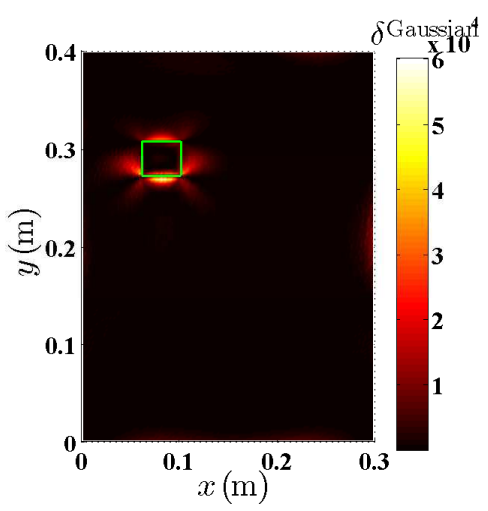

(d)

Fig. 13 (a) Maximum-CDIs, (b) Minimum-CDIs, (c) Mean-CDIs and (d) Gaussian-CDIs associated with noise-free $\mathbf{Z}^{d, 2}$ and the corresponding mode shape from the polynomial fit with $n=8 ; s=0.002 \mathrm{~m}$ in the scheme. 


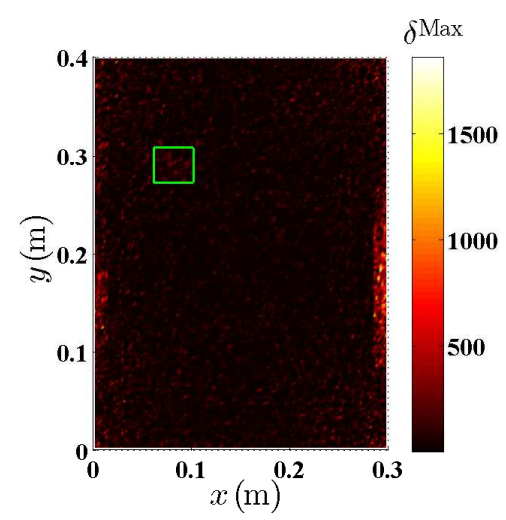

(a)

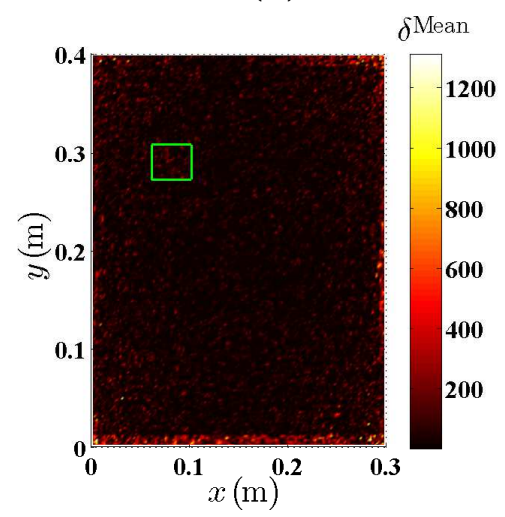

(c)

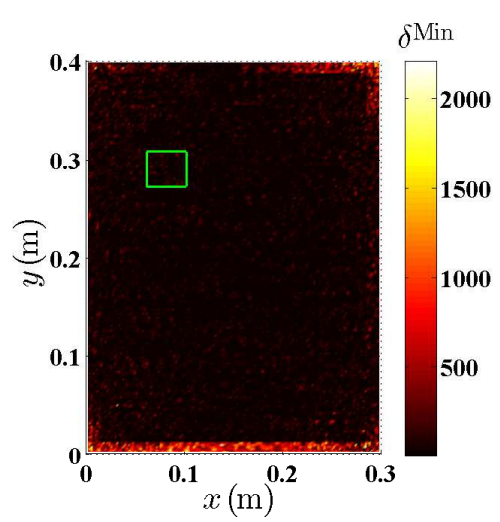

(b)

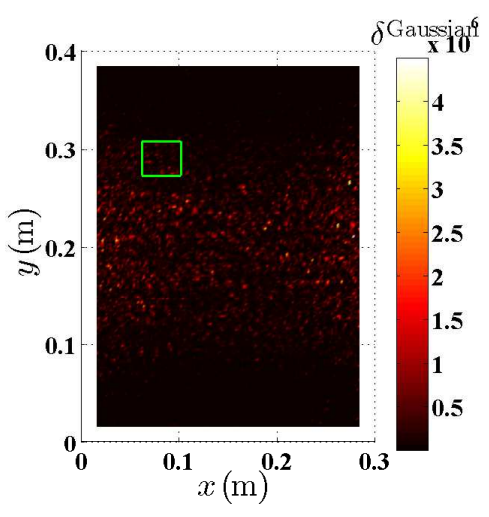

(d)

Fig. 14 (a) Maximum-CDIs, (b) Minimum-CDIs, (c) Mean-CDIs and (d) Gaussian-CDIs associated with $\mathbf{Z}^{d, 2}$ and the corresponding mode shape from the polynomial fit with $n=8 ; s=0.015 \mathrm{~m}$ in the scheme. 


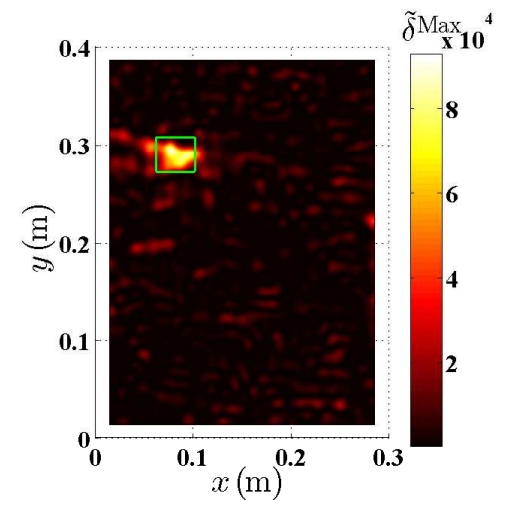

(a)

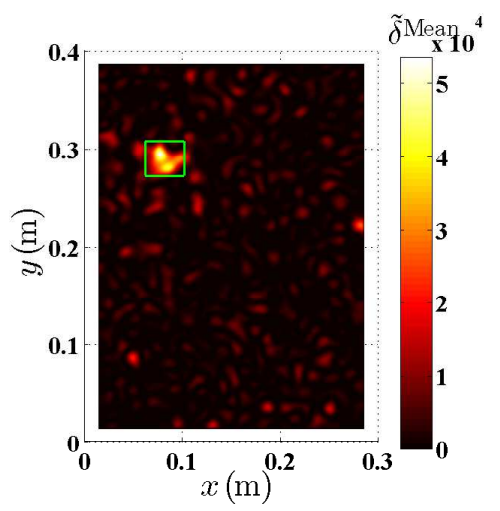

(c)

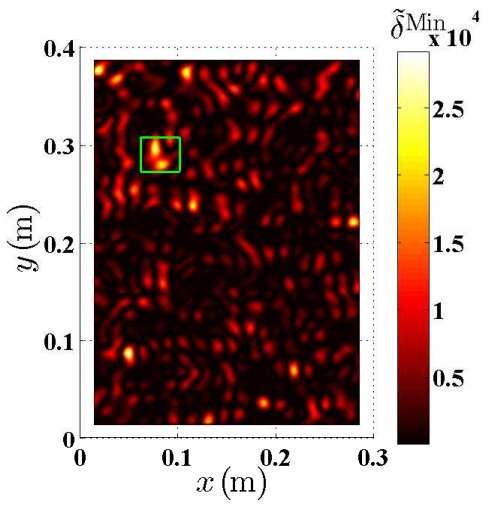

(b)

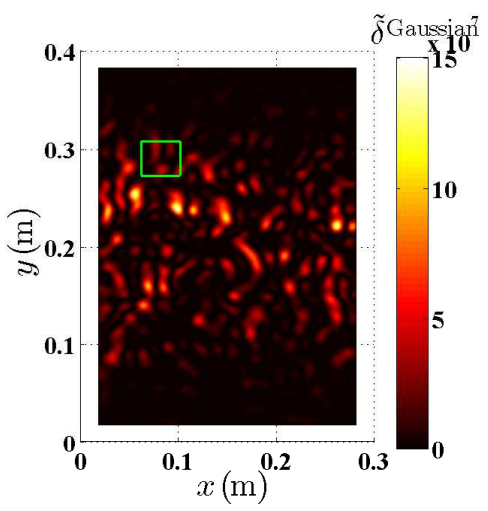

(d)

Fig. 15 (a) Weighted Maximum-CDIs, (b) weighted Minimum-CDIs, (c) weighted Mean-CDIs and (d) weighted Gaussian-CDIs associated with $\mathbf{Z}^{d, 2}$ and the corresponding mode shape from the polynomial fit with $n=8 ; s=0.015 \mathrm{~m}$ in the scheme and $M_{w}=7$ in the weight function. 


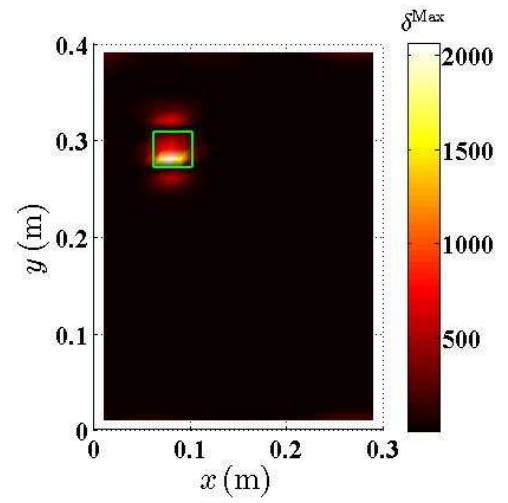

(a)

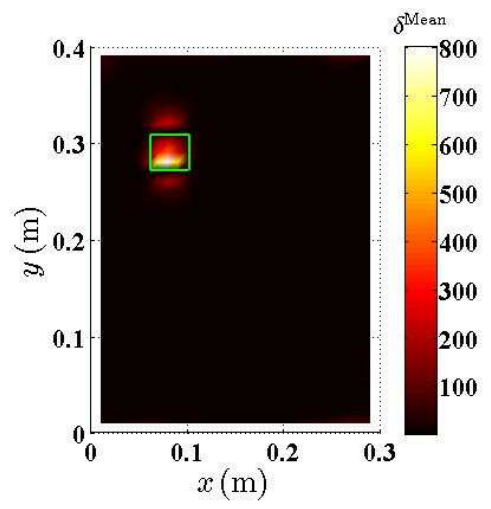

(c)

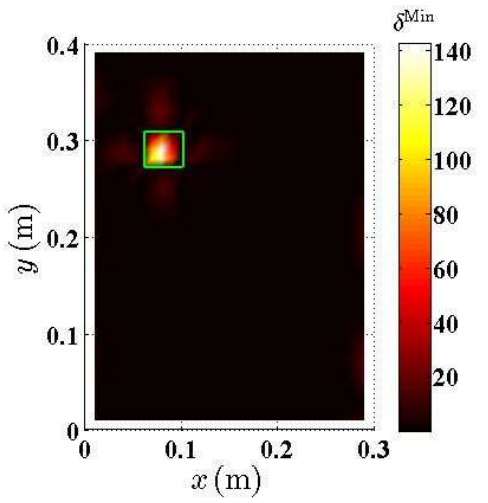

(b)

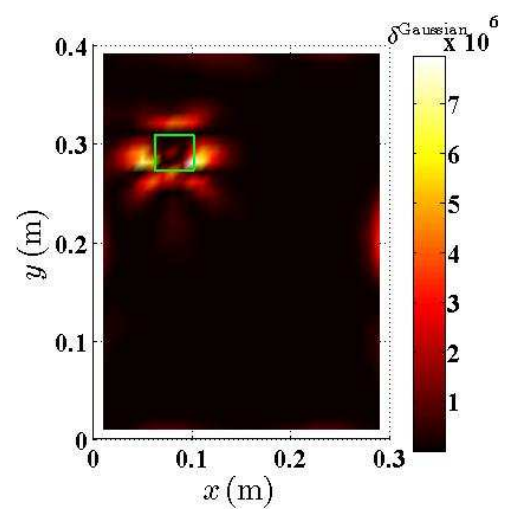

(d)

Fig. 16 (a) Maximum-CDIs, (b) Minimum-CDIs, (c) Mean-CDIs and (d) Gaussian-CDIs associated with noise-free $\mathbf{Z}^{d, 2}$ on the coarse measurement grid and the corresponding mode shape from the polynomial fit with $n=8 ; s=0.002$ $m$ in the scheme. 


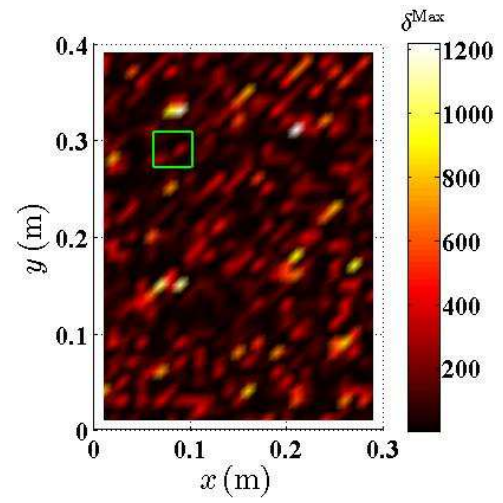

(a)

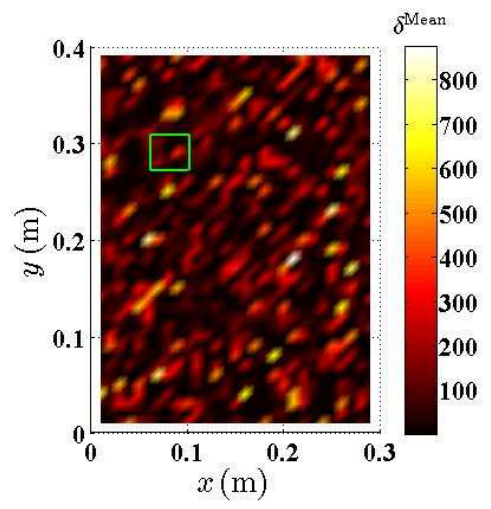

(c)

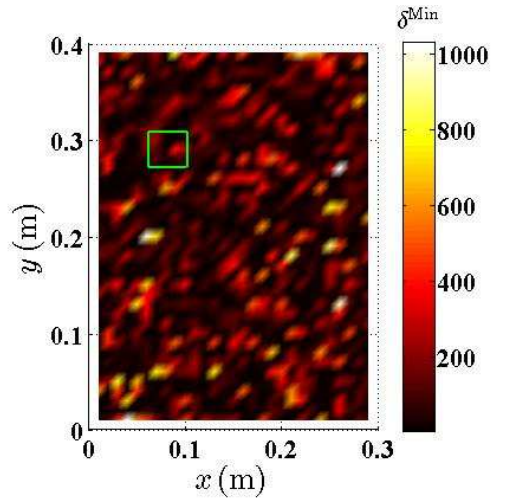

(b)

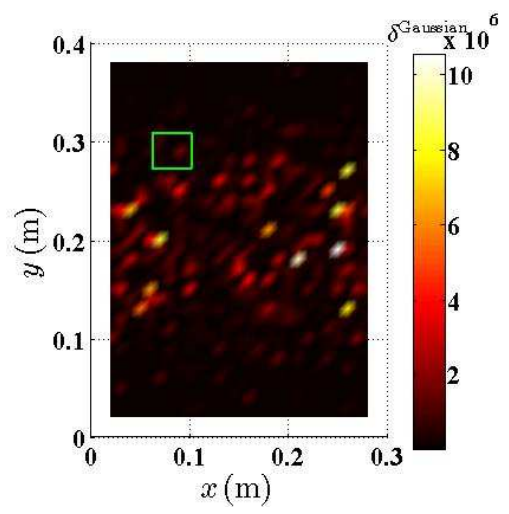

(d)

Fig. 17 (a) Maximum-CDIs, (b) Minimum-CDIs, (c) Mean-CDIs and (d) Gaussian-CDIs associated with $\mathbf{Z}^{d, 2}$ with a signal-to-noise ratio of $60 \mathrm{db}$ on the coarse measurement grid and the corresponding mode shape from the polynomial fit with $n=8 ; s=0.015 \mathrm{~m}$ in the scheme. 


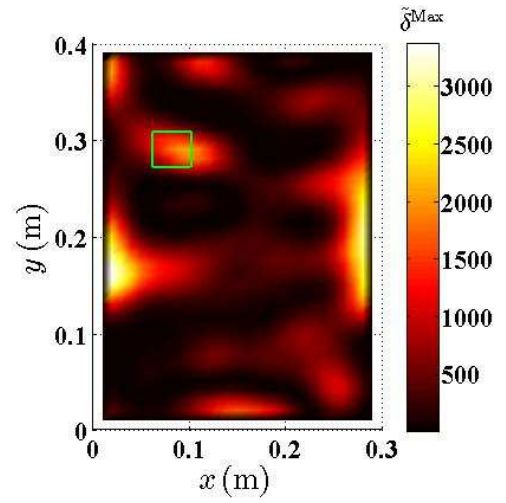

(a)

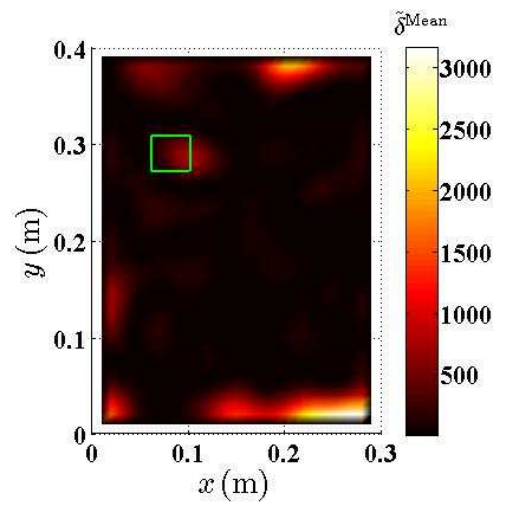

(c)

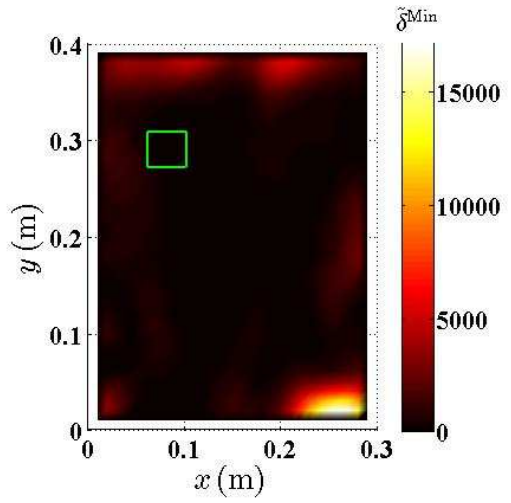

(b)

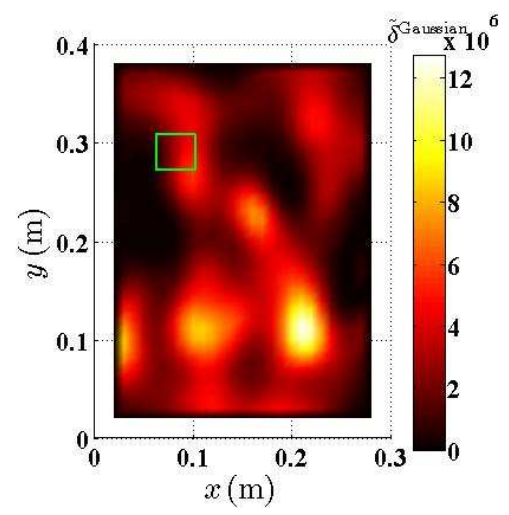

(d)

Fig. 18 (a) Weighted Maximum-CDIs, (b) weighted Minimum-CDIs, (c) weighted Mean-CDIs and (d) weighted Gaussian-CDIs associated with $\mathbf{Z}^{d, 2}$ with a signal-tonoise ratio of $60 \mathrm{db}$ on the coarse measurement grid and the corresponding mode shape from the polynomial fit with $n=8 ; s=0.015 \mathrm{~m}$ in the scheme and $M_{w}=7$ in the weight function. 


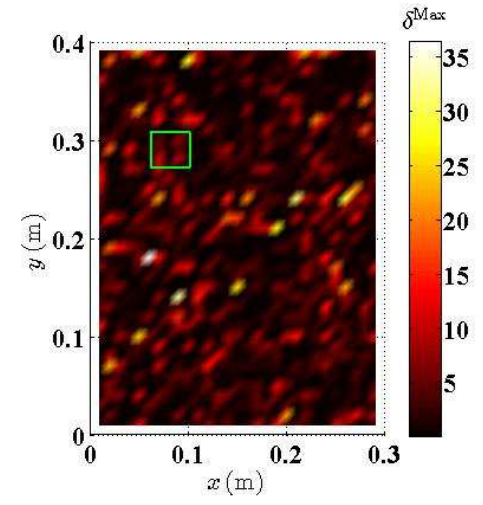

(a)

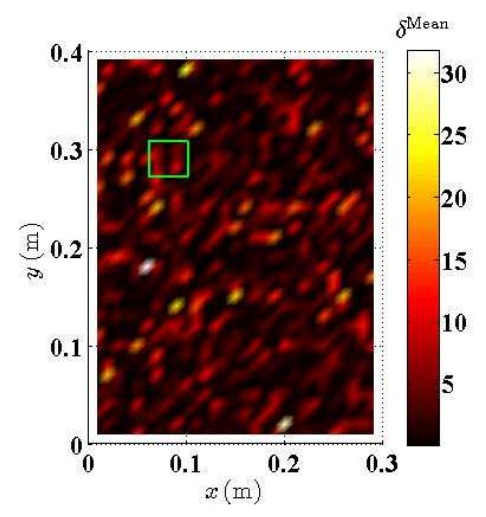

(c)

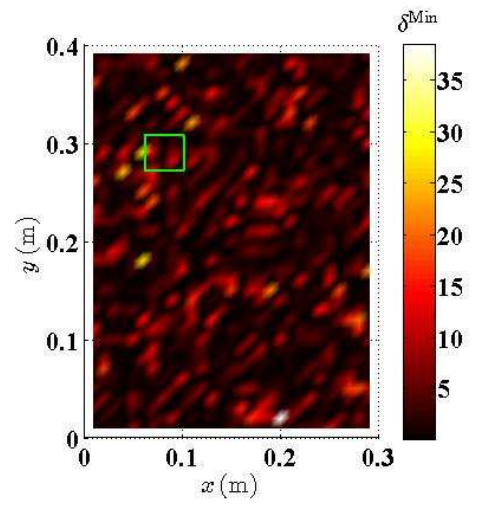

(b)

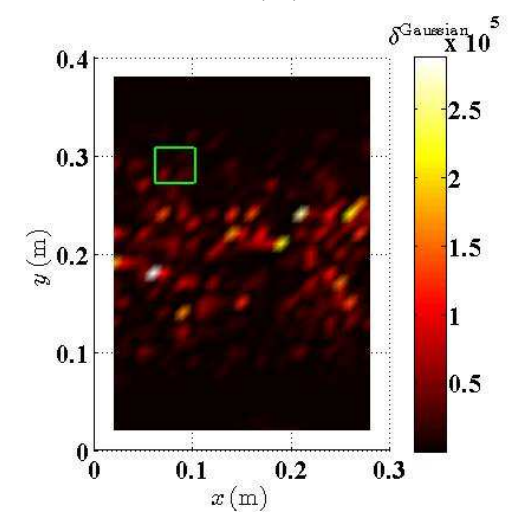

(d)

Fig. 19 (a) Maximum-CDIs, (b) Minimum-CDIs, (c) Mean-CDIs and (d) Gaussian-CDIs associated with $\mathbf{Z}^{d, 2}$ with a signal-to-noise ratio of $75 \mathrm{db}$ on the coarse measurement grid and the corresponding mode shape from the polynomial fit with $n=8 ; s=0.015 \mathrm{~m}$ in the scheme. 


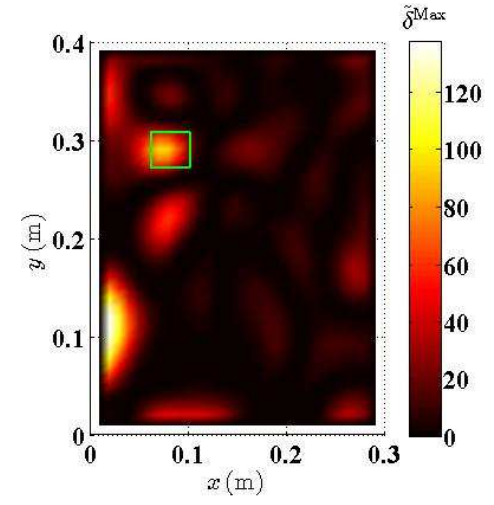

(a)

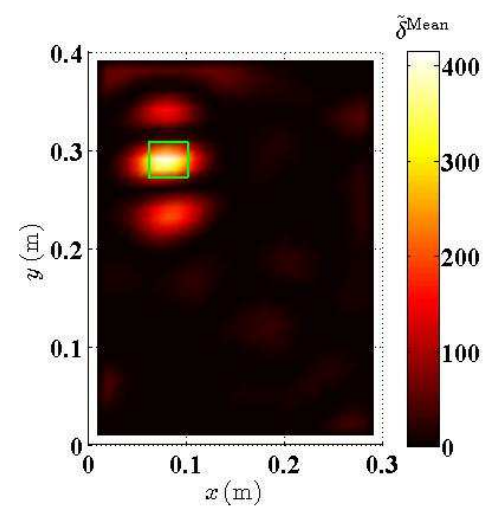

(c)

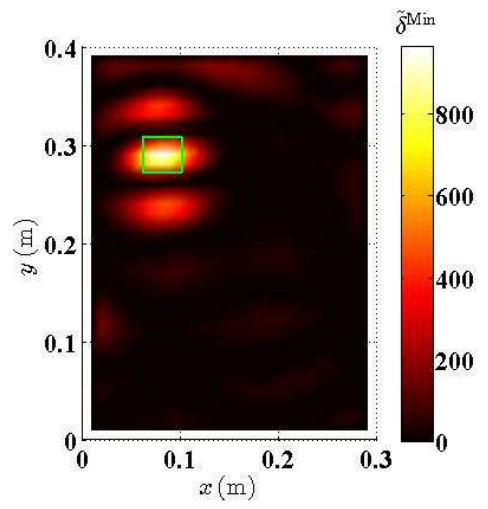

(b)

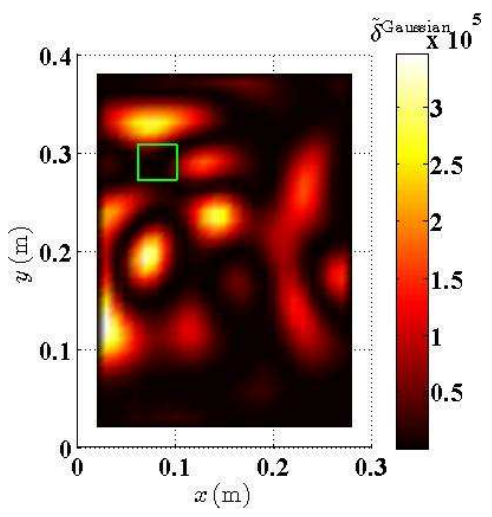

(d)

Fig. 20 (a) Weighted Maximum-CDIs, (b) weighted Minimum-CDIs, (c) weighted Mean-CDIs and (d) weighted Gaussian-CDIs associated with $\mathbf{Z}^{d, 2}$ with a signal-tonoise ratio of $75 \mathrm{db}$ on the coarse measurement grid and the corresponding mode shape from the polynomial fit with $n=8 ; s=0.015 \mathrm{~m}$ in the scheme and $M_{w}=7$ in the weight function. 


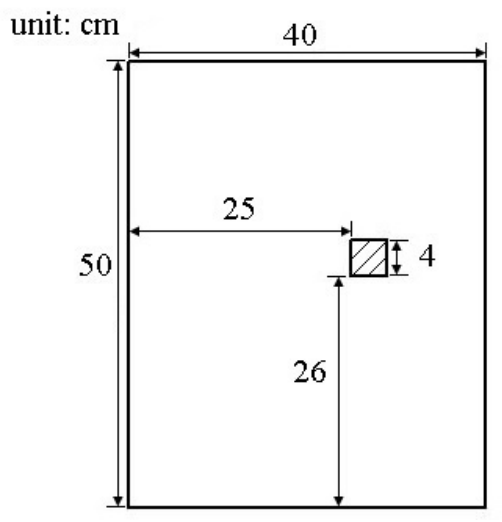

(a)

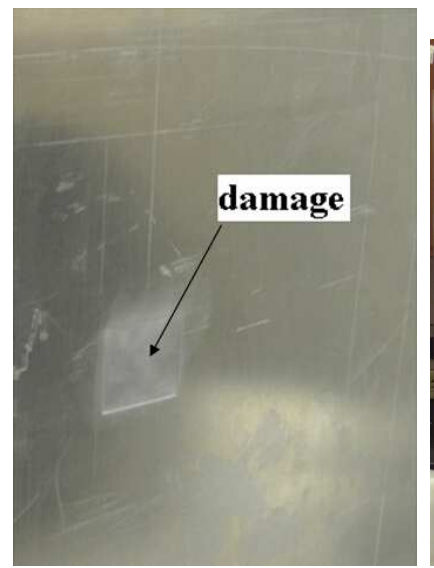

(c)

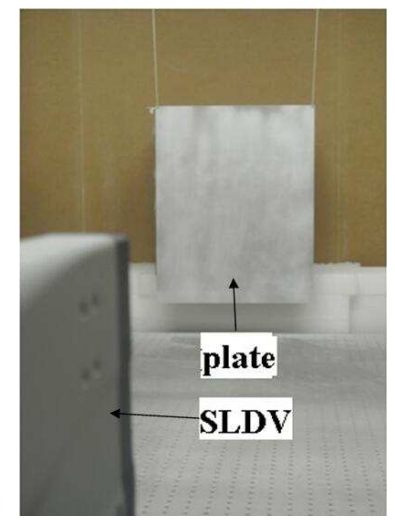

(b)

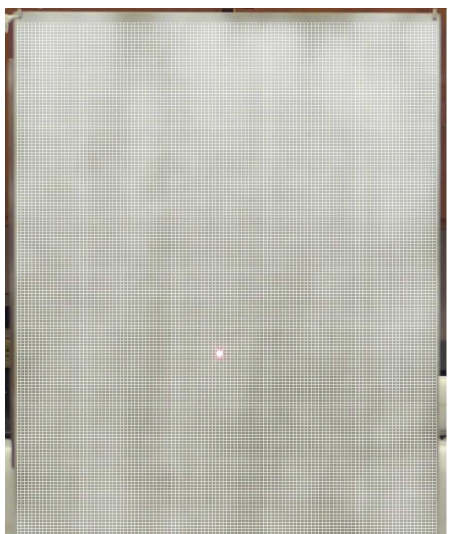

(d)

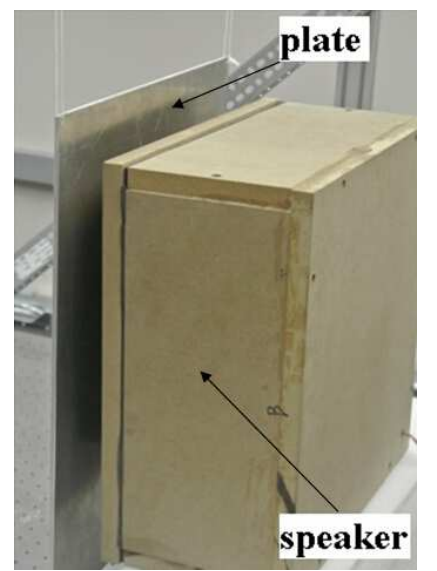

(e)

58

Fig. 21 (a) Dimensions of the damaged plate, (b) the test setup for response measurements of the measurement surface, (c) the damaged surface of the plate, (d) the measurement grid on the measurement surface and (e) the electric speaker facing the damaged surface. Note that SLDV stands for scanning laser Doppler vibrometer in (b). 


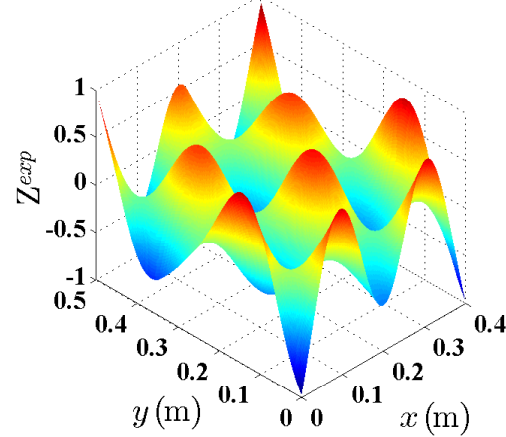

(a)

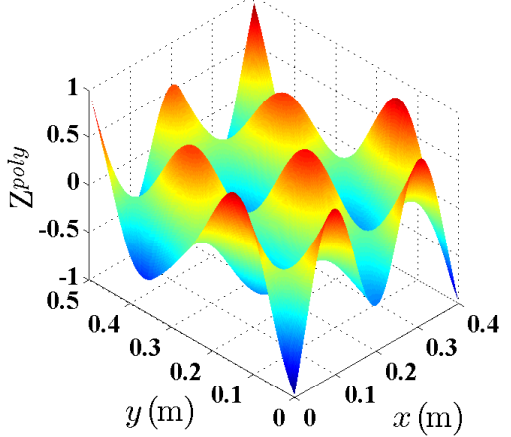

(b)

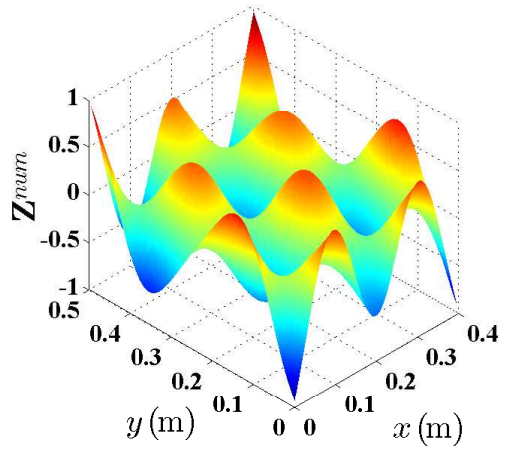

(c)

Fig. 22 (a) Interpolated measured mode shape $\mathbf{Z}^{\exp }$ of the damaged plate at the natural frequency of $1349 \mathrm{~Hz}$, (b) the mode shape from the polynomial that fits $\mathbf{Z}^{e x p}$ with $n=15$ and (c) the mode shape associated with the same mode as that of $\mathbf{Z}^{\exp }$ from a finite element model of the damaged plate. 


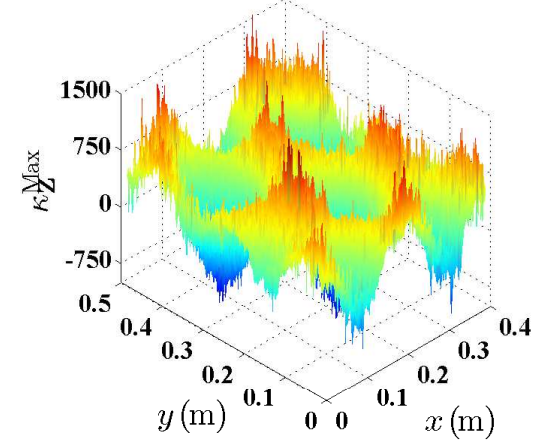

(a)

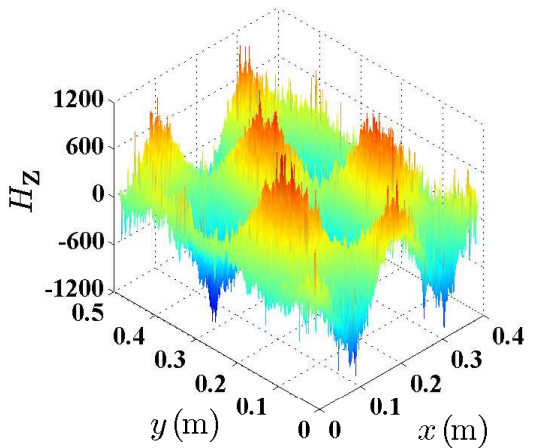

(c)

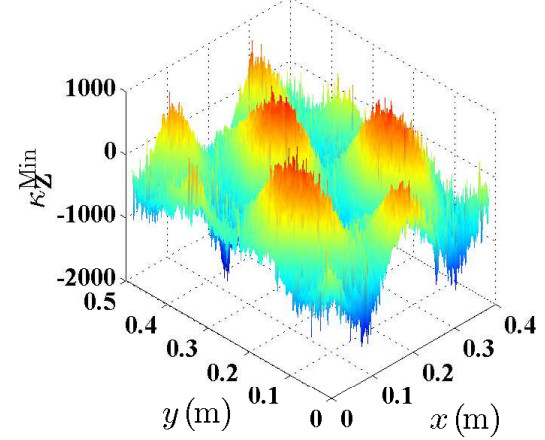

(b)

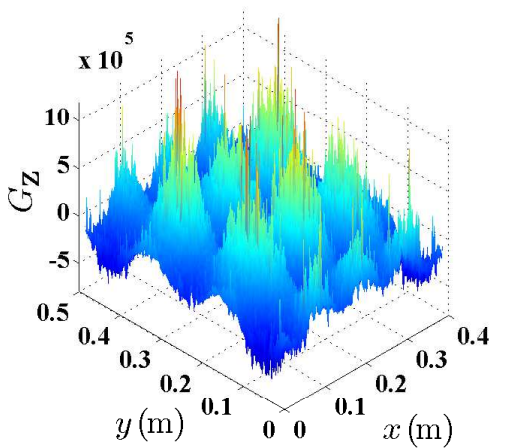

(d)

Fig. 23 (a) Maximum, (b) minimum, (c) mean and (d) Gaussian CMSs associated with $\mathbf{Z}^{\exp }$ from the scheme with $s=0.002 \mathrm{~m}$. 


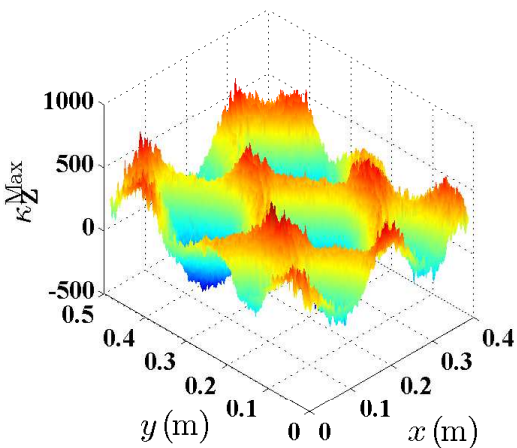

(a)

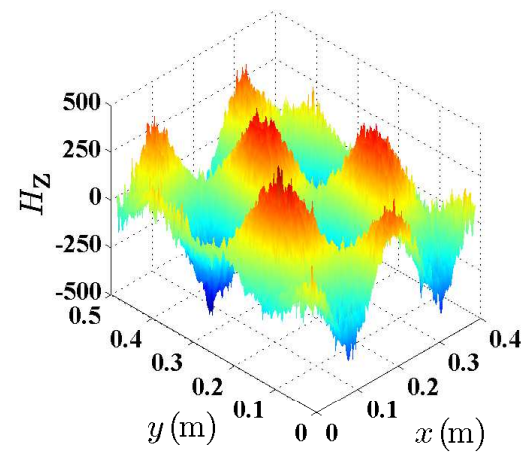

(c)

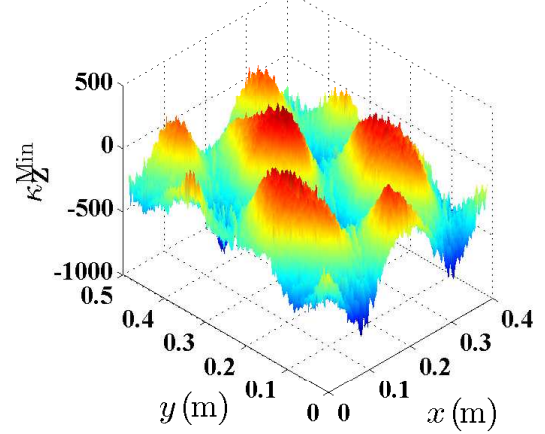

(b)

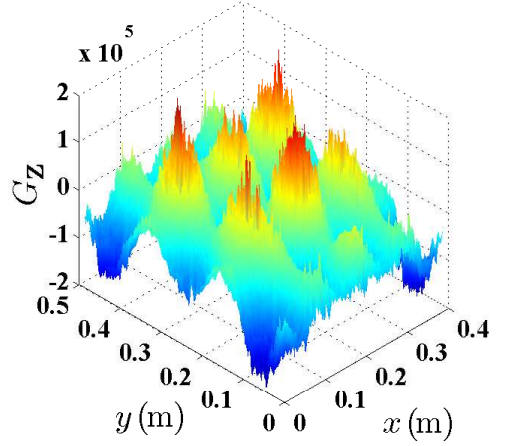

(d)

Fig. 24 (a) Maximum, (b) minimum, (c) mean and (d) Gaussian CMSs associated with $\mathbf{Z}^{\exp }$ from the scheme with $s=0.005 \mathrm{~m}$. 


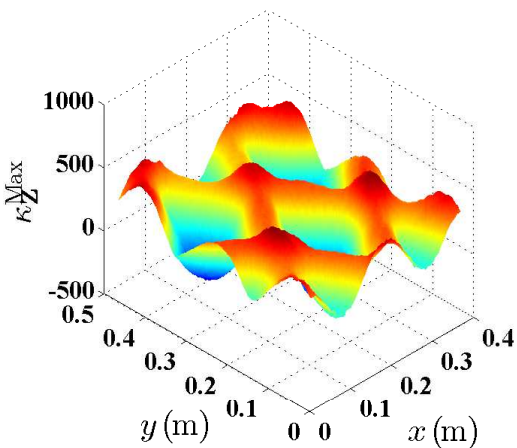

(a)

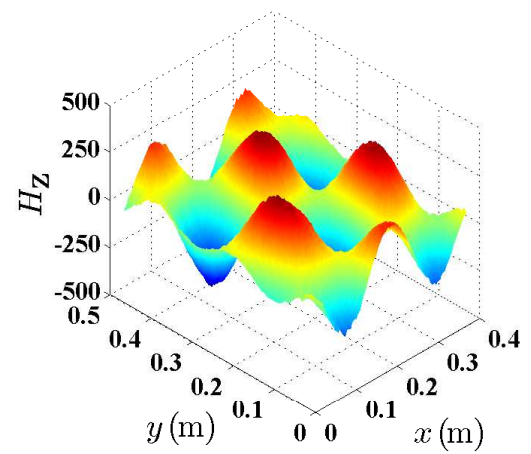

(c)

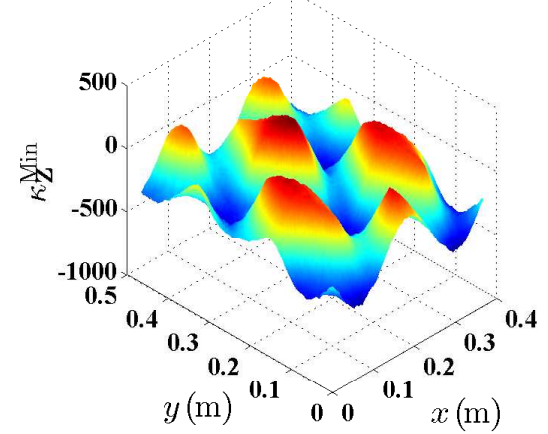

(b)

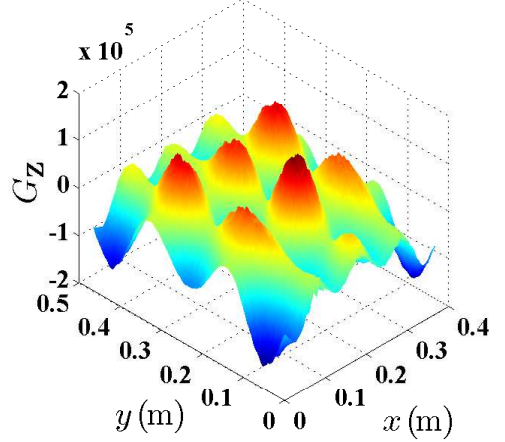

(d)

Fig. 25 (a) Maximum, (b) minimum, (c) mean and (d) Gaussian CMSs associated with $\mathbf{Z}^{\exp }$ from the scheme with $s=0.015 \mathrm{~m}$. 
(a)

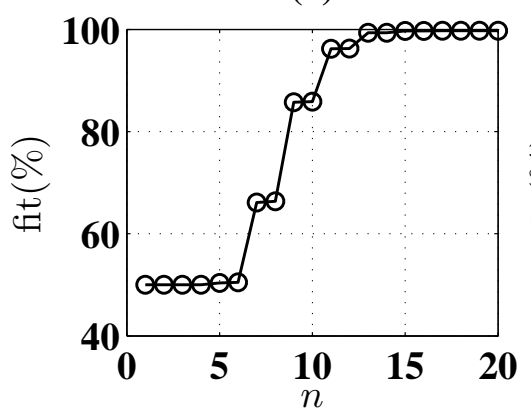

(c)

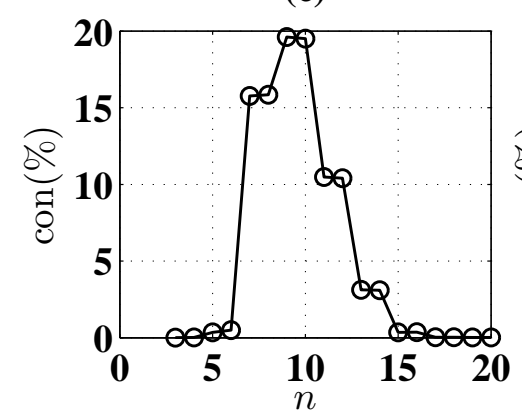

(b)

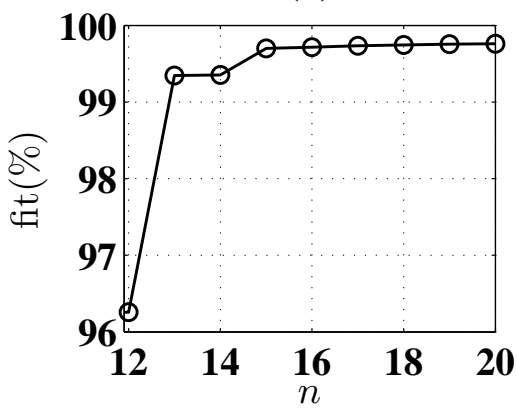

(d)

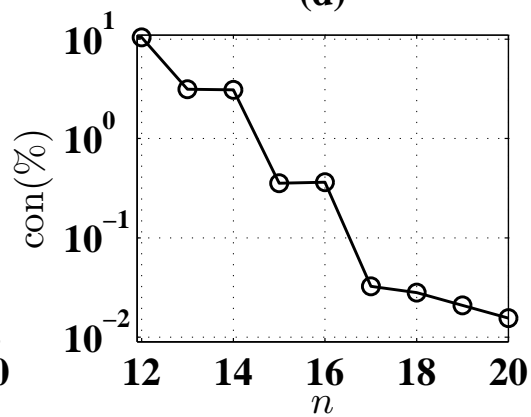

Fig. 26 (a) Fitting indices fit associated with $\mathbf{Z}^{\exp }$ for different $n$, (b) an enlarged view of fit, (c) the convergence indices con associated with $\mathbf{Z}^{\exp }$ for different $n$ and (d) an enlarged view of con. 


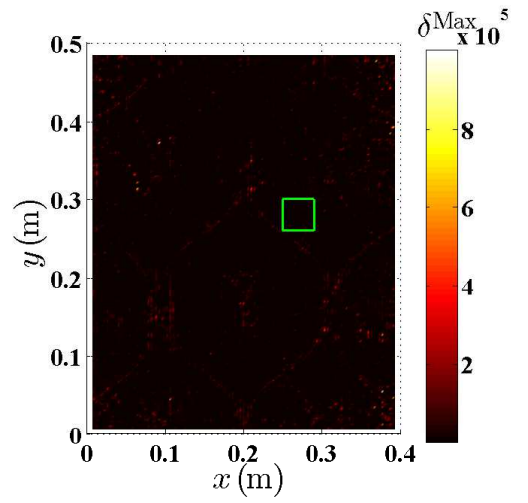

(a)

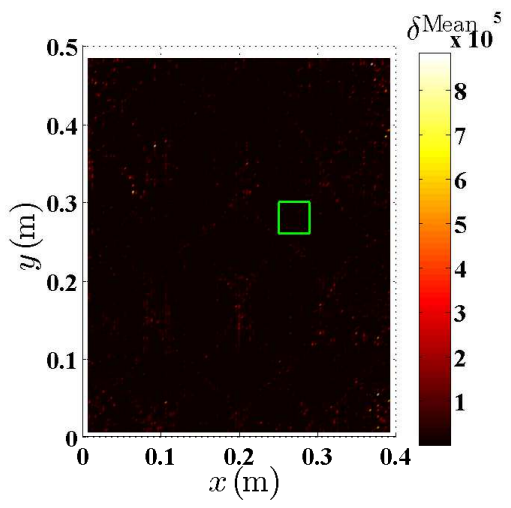

(c)

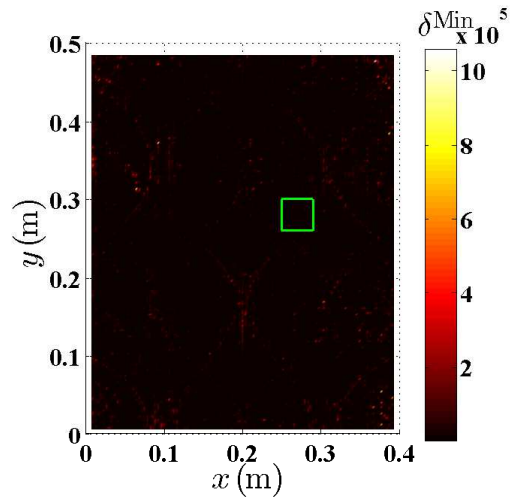

(b)

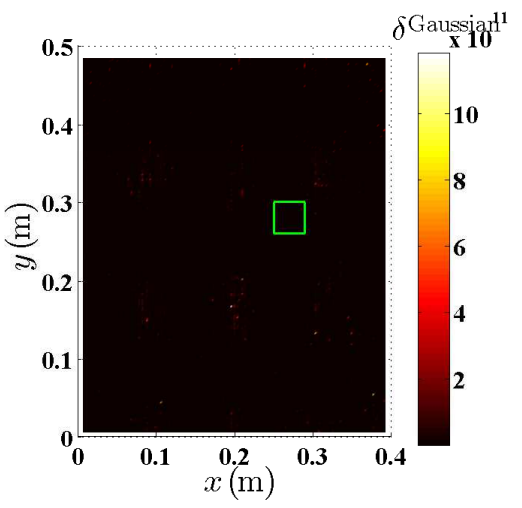

(d)

Fig. 27 (a) Maximum-CDIs, (b) Minimum-CDIs, (c) Mean-CDIs and (d) Gaussian-CDIs associated with $\mathbf{Z}^{\text {exp }}$ and the corresponding mode shape from the polynomial fit with $n=15 ; s=0.002 \mathrm{~m}$ in the scheme. 


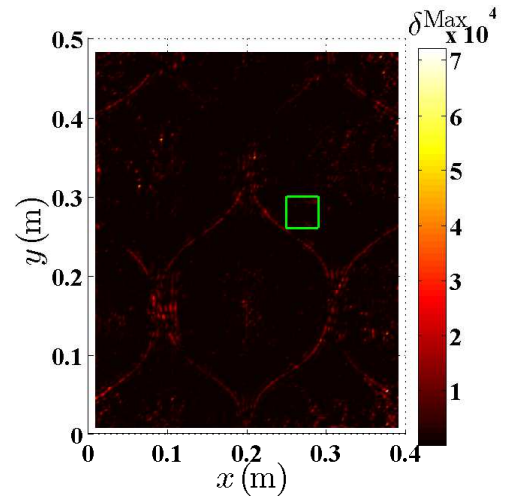

(a)

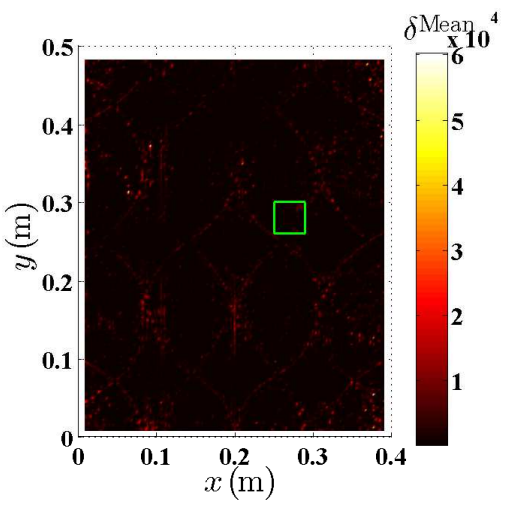

(c)

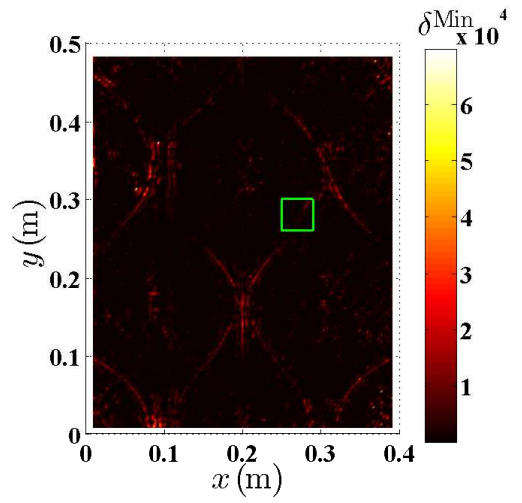

(b)

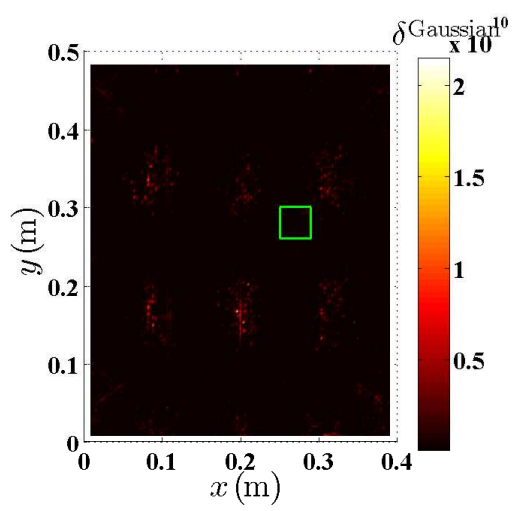

(d)

Fig. 28 (a) Maximum-CDIs, (b) Minimum-CDIs, (c) Mean-CDIs and (d) Gaussian-CDIs associated with $\mathbf{Z}^{\text {exp }}$ and the corresponding mode shape from the polynomial fit with $n=15 ; s=0.005 \mathrm{~m}$ in the scheme. 


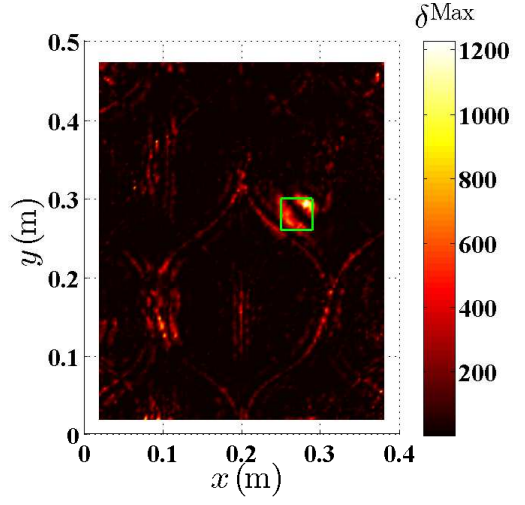

(a)

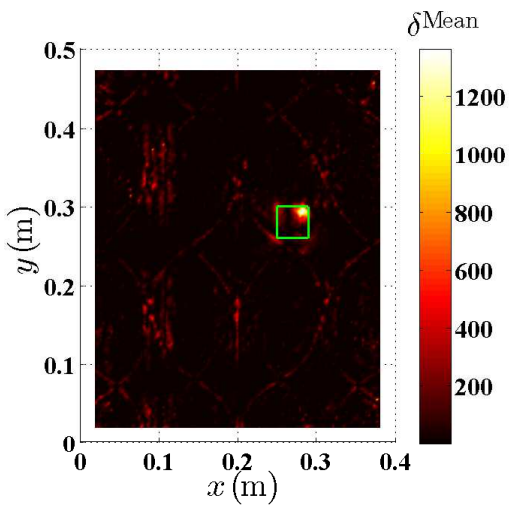

(c)

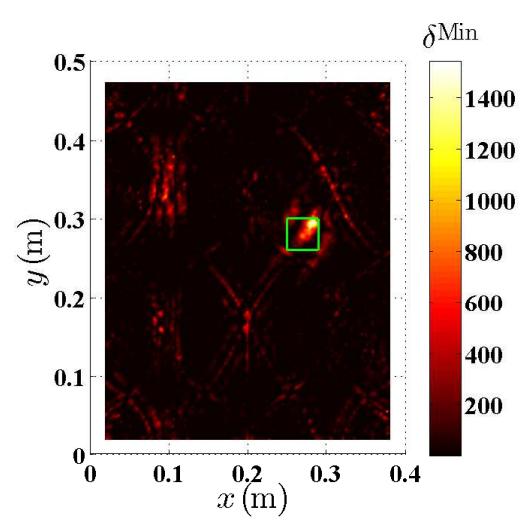

(b)

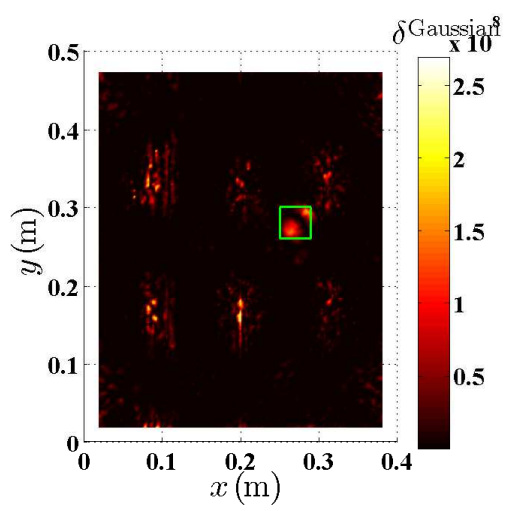

(d)

Fig. 29 (a) Maximum-CDIs, (b) Mininum-CDIs, (c) Mean-CDIs and (d) GaussianCDIs associated with $\mathbf{Z}^{e x p}$ and the corresponding mode shape from the polynomial fit with $n=15 ; s=0.015 \mathrm{~m}$ in the scheme. 


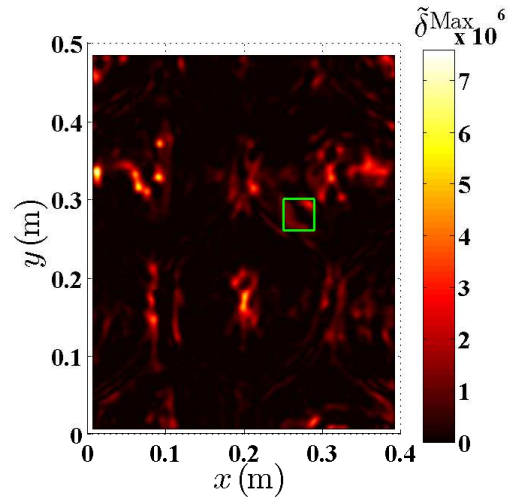

(a)

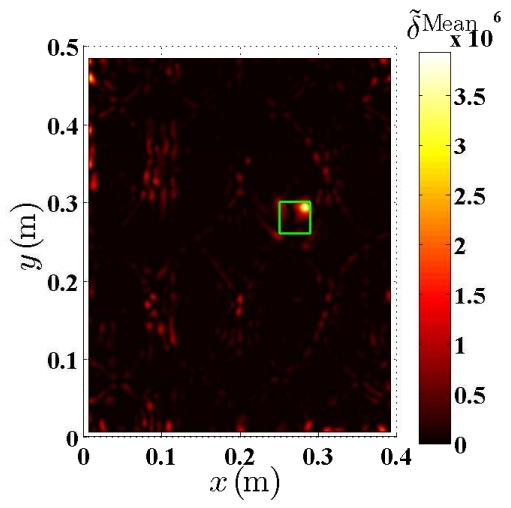

(c)

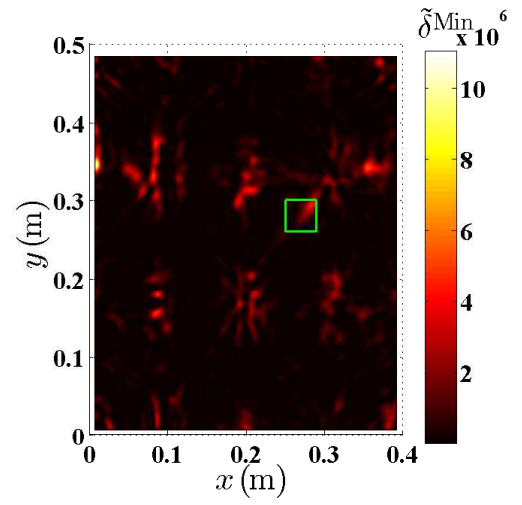

(b)

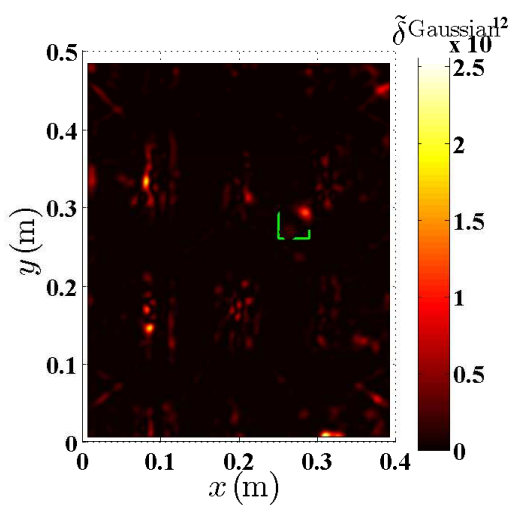

(d)

Fig. 30 (a) Weighted Maximum-CDIs, (b) weighted Minimum-CDIs, (c) weighted Mean-CDIs and (d) weighted Gaussian-CDIs associated with $\mathbf{Z}^{\exp }$ and the corresponding mode shape from the polynomial fit with $n=15 ; s=0.002 \mathrm{~m}$ in the scheme and $M_{w}=7$ in the weight function. 


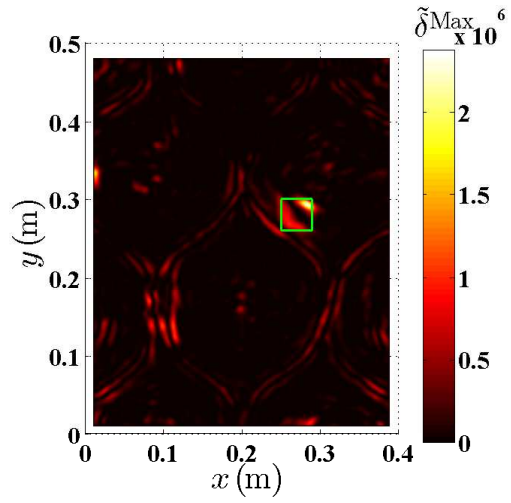

(a)

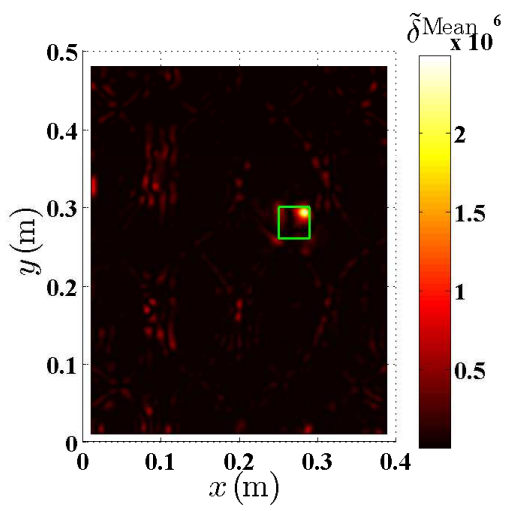

(c)

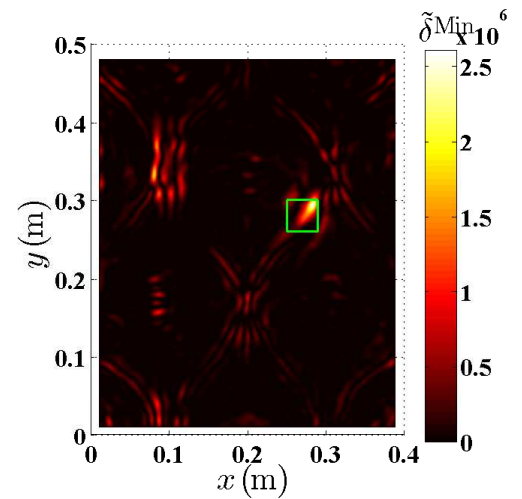

(b)

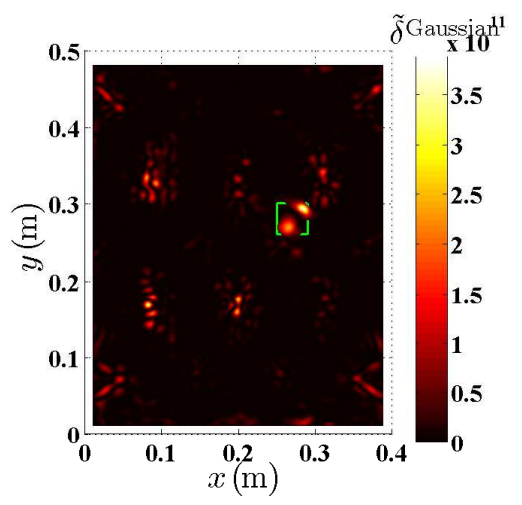

(d)

Fig. 31 (a) Weighted Maximum-CDIs, (b) weighted Minimum-CDIs, (c) weighted Mean-CDIs and (d) weighted Gaussian-CDIs associated with $\mathbf{Z}^{\exp }$ and the corresponding mode shape from the polynomial fit with $n=15 ; s=0.005 \mathrm{~m}$ in the scheme and $M_{w}=7$ in the weight function. 


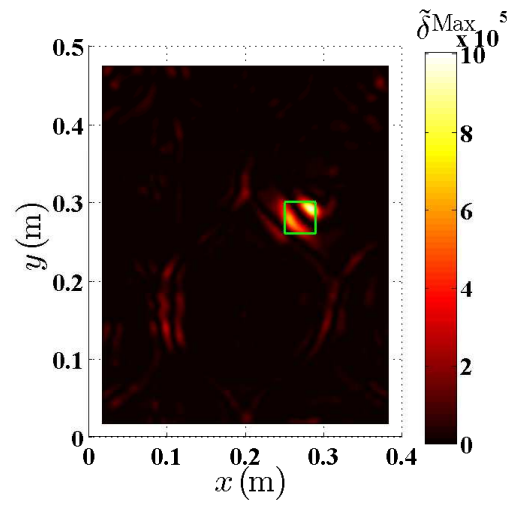

(a)

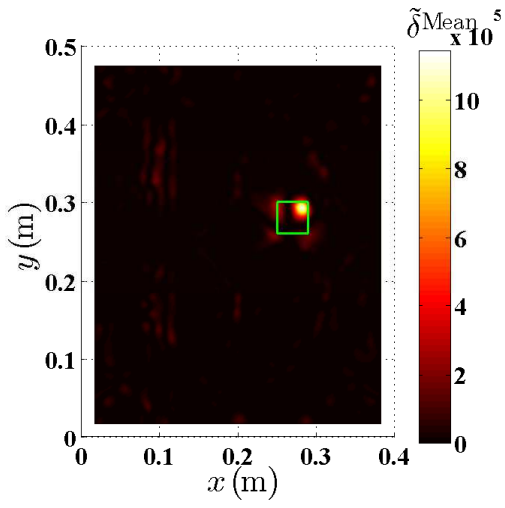

(c)

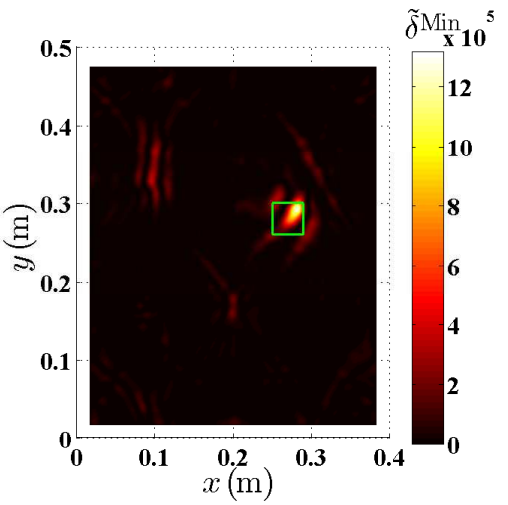

(b)

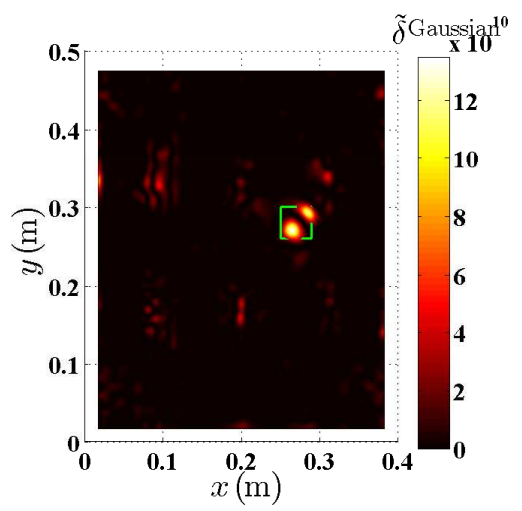

(d)

Fig. 32 (a) Weighted Maximum-CDIs, (b) weighted Minimum-CDIs, (c) weighted Mean-CDIs and (d) weighted Gaussian-CDIs associated with $\mathbf{Z}^{\exp }$ and the corresponding mode shape from the polynomial fit with $n=15 ; s=0.015 \mathrm{~m}$ in the scheme and $M_{w}=7$ in the weight function. 


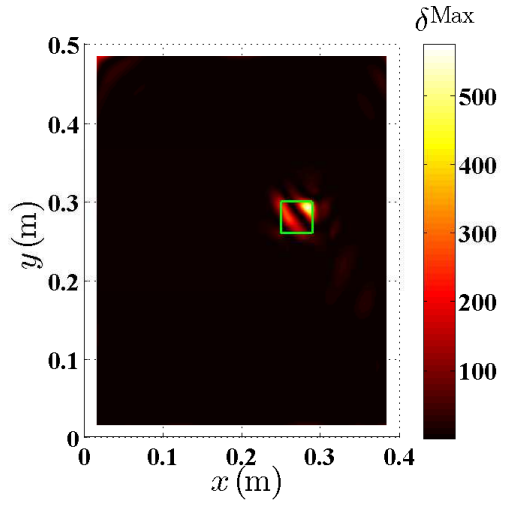

(a)

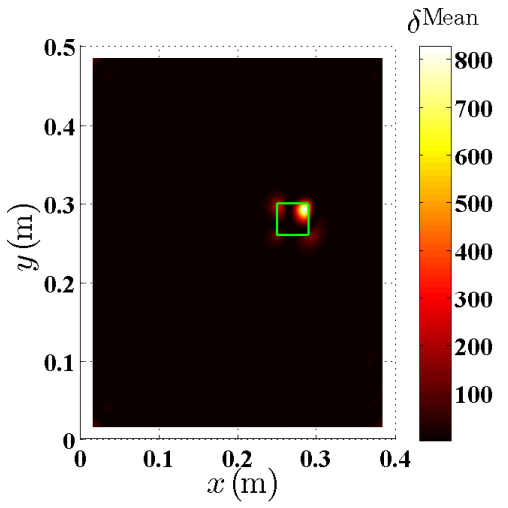

(c)

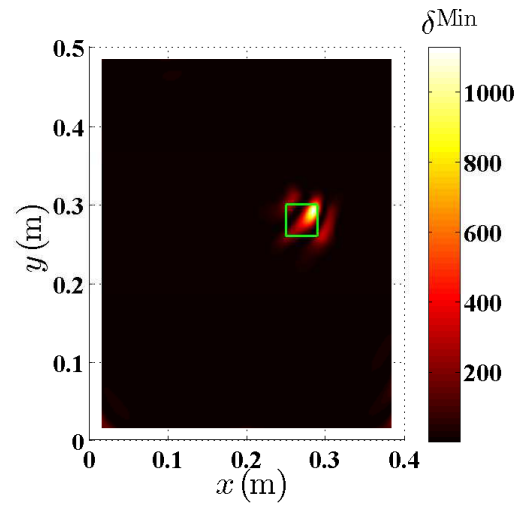

(b)

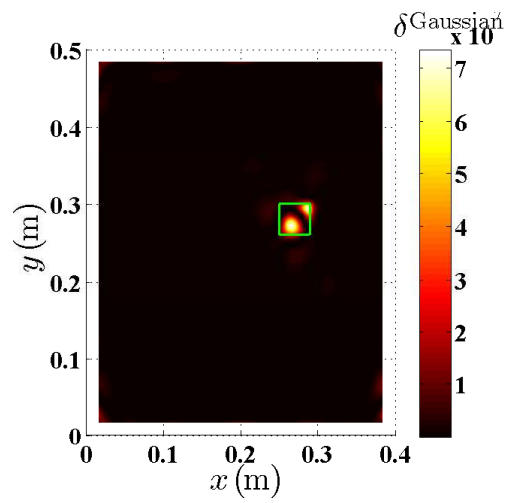

(d)

Fig. 33 (a) Maximum-CDIs, (b) Mininum-CDIs, (c) Mean-CDIs and (d) GaussianCDIs associated with $\mathbf{Z}^{\text {num }}$ and the corresponding mode shape from the polynomial fit with $n=15 ; s=0.015 \mathrm{~m}$ in the scheme. 


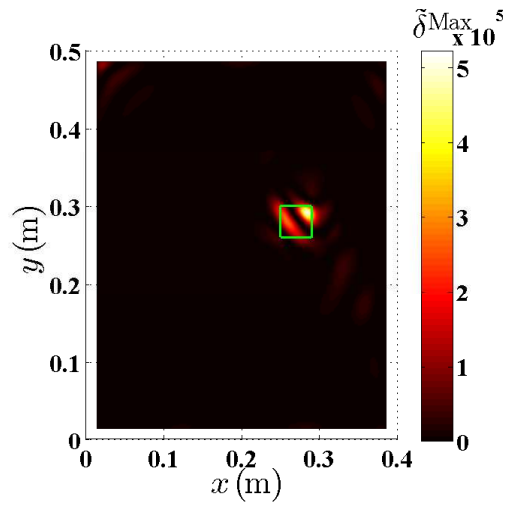

(a)

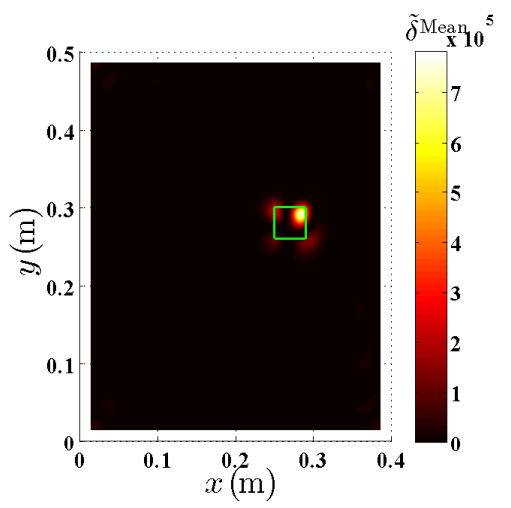

(c)

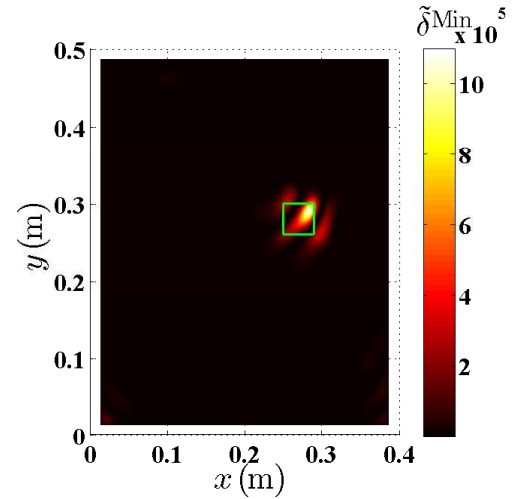

(b)

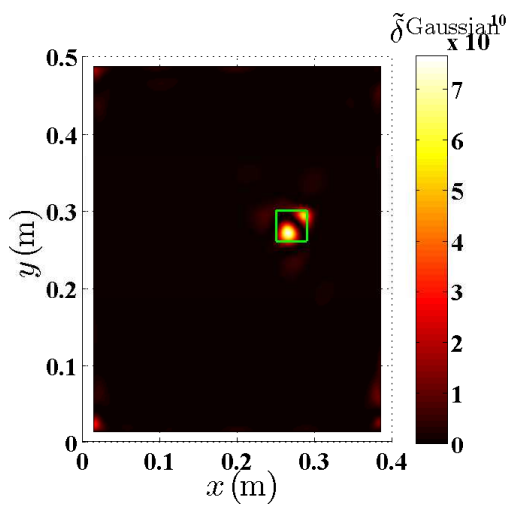

(d)

Fig. 34 (a) Weighted Maximum-CDIs, (b) weighted Minimum-CDIs, (c) weighted Mean-CDIs and (d) weighted Gaussian-CDIs associated with $\mathbf{Z}^{\text {num }}$ and the corresponding mode shape from the polynomial fit with $n=15 ; s=0.015 \mathrm{~m}$ in the scheme and $M_{w}=7$ in the weight function. 